\title{
Stochastic Stability of Event-triggered Anytime Control
}

\author{
Daniel E. Quevedo, Member, IEEE, Vijay Gupta, Member, IEEE, Wann-Jiun \\ Ma, Student Member, IEEE, Serdar Yüksel, Member, IEEE
}

\begin{abstract}
We investigate control of a non-linear process when communication and processing capabilities are limited. The sensor communicates with a controller node through an erasure channel which introduces i.i.d. packet dropouts. Processor availability for control is random and, at times, insufficient to calculate plant inputs. To make efficient use of communication and processing resources, the sensor only transmits when the plant state lies outside a bounded target set. Control calculations are triggered by the received data. If a plant state measurement is successfully received and while the processor is available for control, the algorithm recursively calculates a sequence of tentative plant inputs, which are stored in a buffer for potential future use. This safeguards for time-steps when the processor is unavailable for control. We derive sufficient conditions on system parameters for stochastic stability of the closed loop and illustrate performance gains through numerical studies.
\end{abstract}

\section{INTRODUCTION}

Due to the tight coupling among the cyber and the physical cores in many cyber-physical systems, it is imperative to develop systematic design principles for controllers with limited communication and processing resources. Both the areas of control with communication constraints

D. Quevedo is with the School of Electrical Engineering \& Computer Science, The University of Newcastle, Australia, dquevedo@ieee.org. V. Gupta and W. J. Ma are with the Department of Electrical Engineering, University of Notre Dame, USA, vgupta2@nd.edu, wma1@nd.edu. S. Yüksel is with the Department of Mathematics and Statistics, Queen's University, Kingston, ON K7L 3N6 Canada, yuksel@mast.queensu.ca. Research supported for the first author under Australian Research Council's Discovery Projects funding scheme (project number DP0988601) and in part for the second and third authors by NSF awards 0846631 and 0834771. 


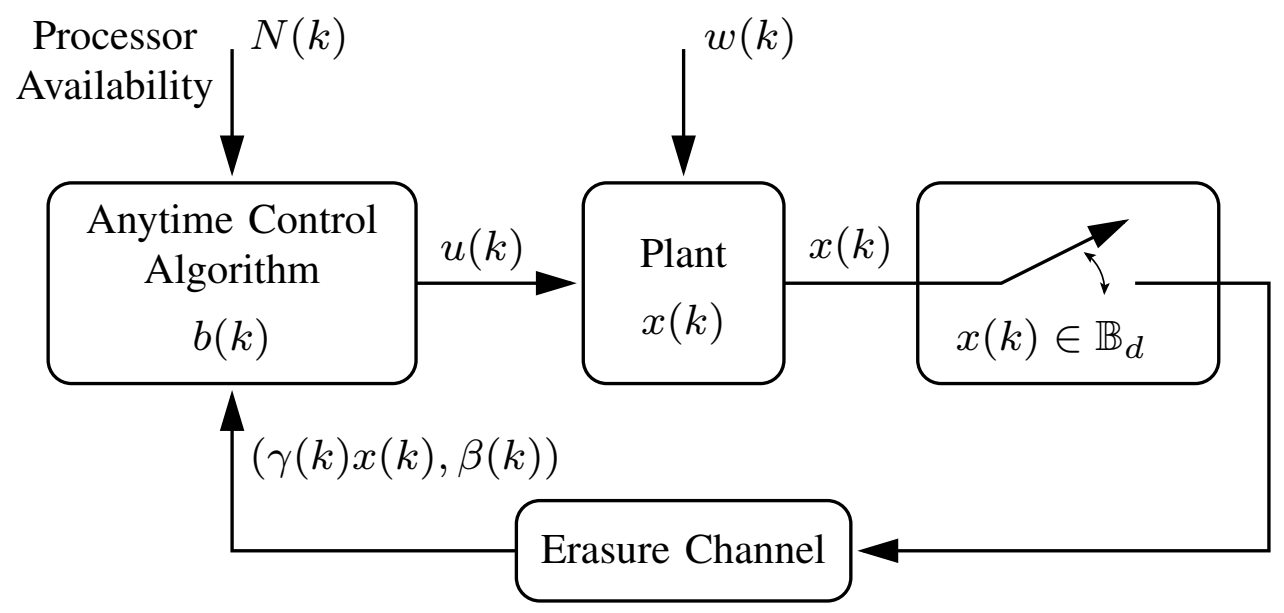

Fig. 1. Event-triggered Anytime Control with Unreliable Communications.

and control with limited and time-varying processor availability have accordingly received much attention.

Control design in the presence of practical communication channels and protocols has been studied in the area of networked control systems. Of particular interest to the present work is the literature on control across analog erasure channels; see, e.g., [1]-[4]. Apart from arising from data transmission across a wireless channel, data loss may also arise due to congestion in a communication network, possibly transmitted by a control loop. To minimize this source of data loss, one can conceive event triggered communication schemes in which sensors transmit information only if the system state exceeds a certain bound; see, e.g., [5]-[9]. Recently, work has also been done on designing event triggering rules to ensure stability in the face of data dropouts. However, most works are restricted to single integrator dynamics [10], [11] or are numerical studies [12].

On the other hand, various works have also considered the impact of limited or time-varying processing power on closed-loop control [13]-[15]. Interestingly, event-triggered and self-triggered updates of the control inputs have also been proposed to ensure less demand on the processor on average by calculating the control input on demand [6], [16]. The direction of anytime control has also shown promise [17]-[20]. Such algorithms calculate a coarse control input even with limited processing resources and refine the input as more processing resources become available. 
The quality of control inputs is thus time-varying, but no control input is obtained only rarely.

Notwithstanding the advances discussed above, relatively few works have considered control design under both limited communication and processing resources. Optimal control design for arbitrary non-linear processes under communication and processing constraints is likely a challenging problem, since certainty equivalence would not hold in general [21]. Accordingly, in the present note we consider a pre-designed control law, and focus on the implementation of this controller in the presence of both communication and processing limitations. As depicted in Fig. 1, we consider a discrete-time non-linear plant being controlled across a communication network that stochastically erases data transmitted across it. To reduce congestion in the network, the sensor employs an event triggered communication strategy. However, due to time-varying availability of the processing resources, it is not guaranteed that the processor can calculate a control input at all time steps when the sensor transmits (even if the network does not erase the data). To maximally utilize the processing resources, the controller employs an anytime control algorithm. Under such a setting, we analyze stochastic stability of the closed loop. Our main stability results are stated in terms of an inequality that relates open-loop growth of the plant state, packet erasure probability, and parameters of the processor availability model. For the particular case where processing resources are available at every time step, our result reduces to a sufficient condition for stochastic stability of non-linear control in where sensor communicates according to an event-triggering condition across an analog i.i.d. erasure link. This may be of independent interest.

Notation: We write $\mathbb{N}$ for $\{1,2, \ldots\}$ and $\mathbb{N}_{0}$ for $\mathbb{N} \cup\{0\}$. $\mathbb{R}$ represents the real numbers and $\mathbb{R}_{\geq 0} \triangleq[0, \infty)$. The $p \times p$ identity matrix is denoted via $I_{p}, 0_{p \times q}$ is the $p \times q$ all-zeroes matrix, $0_{p} \triangleq 0_{p \times p}$, and $\mathbf{0}_{p} \triangleq 0_{p \times 1}$. The notation $\{x\}_{\mathcal{K}}$ stands for $\{x(k): k \in \mathcal{K}\}$, where $\mathcal{K} \subseteq \mathbb{N}_{0}$. We adopt the conventions $\sum_{k=\ell_{1}}^{\ell_{2}} a_{k}=0$ and $\prod_{k=\ell_{1}}^{\ell_{2}} a_{k}=1$, if $\ell_{1}>\ell_{2}$ and irrespective of $a_{k} \in \mathbb{R}$. The superscript ${ }^{T}$ refers to transpose. The Euclidean norm of a vector $x$ is denoted via $|x|=\sqrt{x^{T} x}$. A function $\varphi: \mathbb{R}_{\geq 0} \rightarrow \mathbb{R}_{\geq 0}$ is of class- $\mathscr{K}_{\infty}\left(\varphi \in \mathscr{K}_{\infty}\right)$, if it is continuous, zero at zero, strictly increasing, and unbounded. The probability of an event $\Omega$ is denoted by $\operatorname{Pr}\{\Omega\}$ and the conditional probability of $\Omega$ given $\Gamma$ by $\operatorname{Pr}\{\Omega \mid \Gamma\}$. The expected value of a random variable $x$ given $\Gamma$ is denoted by $\mathbf{E}\{x \mid \Gamma\}$, while $\mathbf{E}\{x\}$ refers to the unconditional expectation. The expression $x \sim \nu$ denotes that the random variable $x$ has probability distribution $\nu$ and $\mathbf{E}_{\nu}\{x\}$ denotes the expectation under probability distribution $\nu$. 


\section{EVEnT-DRIVEn CONTROL OVER AN ERASURE CHANNEL}

We consider non-linear (and possibly open-loop unstable) plants, sampled periodically with sampling interval $T_{s}>0$ (see Fig. 1),

$$
x(k+1)=f(x(k), u(k)), \quad k \in \mathbb{N}_{0},
$$

where $x \in \mathbb{R}^{n}$ is the plant state, and $u \in \mathbb{U} \subseteq \mathbb{R}^{p}$ with $\mathbf{0}_{p} \in \mathbb{U}$ is the (possibly constrained) plant input. The initial state $x(0)$ is arbitrarily distributed. The plant is equipped with a sensor, which has direct access to the plant state at the sampling instants $k \in \mathbb{N}_{0}$.

To save on communication expenditure, the sensor adopts an event-triggered transmission strategy, in which the sensor transmits only at instances $k \in \mathbb{N}_{0}$, where $x(k) \notin \mathbb{B}_{d} \triangleq\{x \in$ $\left.\mathbb{R}^{n}:|x|<d\right\}$. This transmission is across an erasure channel which introduces random packet dropouts. To keep communication costs low, the controller does not send acknowledgments back to the sensor and no re-transmissions are allowed. We introduce two discrete random processes, namely $\{\gamma\}_{\mathbb{N}_{0}}$ and $\{\beta\}_{\mathbb{N}_{0}}$. The binary transmission success process $\{\gamma\}_{\mathbb{N}_{0}}$ describes packet loss: a successful transmission at time $k$ is denoted by $\gamma(k)=1$ and a packet erasure by $\gamma(k)=0$. The ternary process $\{\beta\}_{\mathbb{N}_{0}}$ incorporates the event-based transmission rule,

$$
\beta(k)= \begin{cases}\gamma(k) & \text { if the sensor transmitted at time } k, \\ 2 & \text { if the sensor did not transmit at time } k .\end{cases}
$$

Thus, $\beta(k)=2 \Leftrightarrow|x(k)|<d$. We assume that $\beta(k)$ is known to the controller at time $k$ through monitoring of received energy in the sensor transmission band. Transmission outcomes trigger the functions carried out by the controller. The scalar $d \in \mathbb{R}_{\geq 0}$ is a design parameter, which determines communication channel utilization and control performance. Elucidating the trade-off between these quantities is one of the motivations of the present work.

When implementing discrete-time control systems, it is generally assumed that the processing resources available to the controller are such that the desired control law can be evaluated within a fixed time-delay, say $\delta \in\left(0, T_{s}\right)$. However, in practical networked and embedded systems, the processing resources available for control calculations may vary and, at times, be insufficient to generate a control input within the prescribed time-delay $\delta$ [15]. In the sequel we will further develop our anytime control algorithm of [20], [22] to seek favorable trade-offs between processor 
and communication availability, and control performance. We will assume that the plant model (1) is globally stabilizable via state feedback.

Assumption 1 (Stabilizability): There exist $V: \mathbb{R}^{n} \rightarrow \mathbb{R}_{\geq 0}, \varphi_{1}, \varphi_{2} \in \mathscr{K}_{\infty}, \kappa: \mathbb{R}^{n} \rightarrow \mathbb{U}$, and a constant $\rho \in[0,1)$, such that

$$
\begin{array}{cc}
\varphi_{1}(|x|) \leq V(x) \leq \varphi_{2}(|x|), & \forall x \in \mathbb{R}^{n}, \\
V(f(x, \kappa(x))) \leq \rho V(x), & \forall x \notin \mathbb{B}_{d} .
\end{array}
$$

To encompass processing constraints, we will assume that the controller needs processor time to carry out mathematical computations, such as evaluating $\kappa$. However, input-output operations and simple operations at a bit level, e.g., writing data into buffers, shifting buffer contents and setting values to zero, do not require processor time.

Before proceeding we note that a direct implementation of $\kappa$ used in Assumption 1, when processing resources are time varying, sensor transmissions are event-triggered, and the sensor transmissions are affected by dropouts, results in the baseline event-based algorithm

$$
u(k)= \begin{cases}\kappa(x(k)) & \text { if } \beta(k)=1 \text { and processor is available, } \\ \mathbf{0}_{p} & \text { otherwise, }\end{cases}
$$

where the symbol $u(k)$ with $k \in \mathbb{N}_{0}$ denotes the plant input which is applied during the interval $\left[k T_{s}+\delta,(k+1) T_{s}+\delta\right)$. Whilst the baseline algorithm is intuitive, our previous works [20], [22] suggest that it will be outperformed by more elaborate control formulations.

\section{Event-Driven Anytime Control Algorithm}

The anytime algorithm is based on the following idea: control calculations are triggered whenever a new measurement is successfully received. However, the precise number of control inputs calculated depends on the processing resources available. At time intervals when the controller is provided with more processing resources than are needed to evaluate the current control input, the algorithm calculates a sequence of tentative future plant inputs. The sequence is stored in a local buffer and may be used when, at some future time steps, the processor availability precludes any control calculations even though new state information is received.

In our recent work [20], [22], we analyzed this algorithm for the simpler case where the controller has direct access to plant state $x(k)$ at all instants $k \in \mathbb{N}_{0}$. In the present work we alleviate this assumption by considering that sensor transmissions are event-triggered and through 


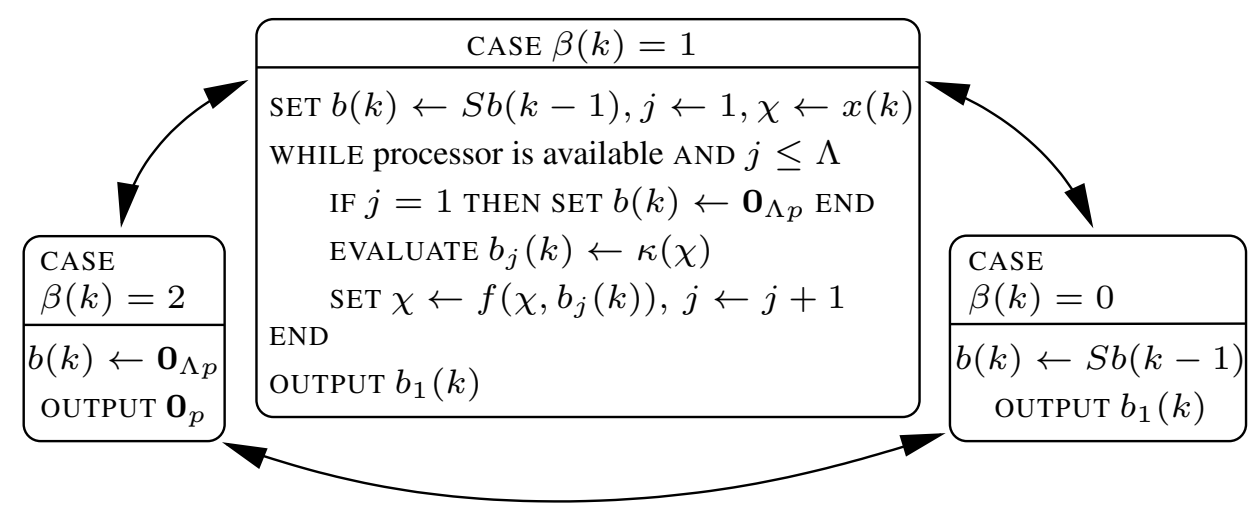

Fig. 2. Operating modes of anytime Algorithm $\mathrm{A}_{1}$ during the time interval $\left[k T_{s},(k+1) T_{s}\right)$.

a communication channel which introduces random dropouts. In addition, to save energy and processing resources, the controller is event-triggered. More precisely, the actions taken by the controller are guided by the value of $\beta(k)$ and the processor availability.

If $\beta(k)=1$, then the controller uses $x(k)$ to calculate tentative control values, provided the processor is available for control. This sequence will be stored in a buffer. If the processor is not available or $\beta(k)=0$, then the controller does not do any calculations and the plant input is provided by previously calculated buffered values (if available). The instances $\beta(k)=2$ refer to situations where the plant state is at the desired region $\mathbb{B}_{d}$, and $x(k)$ is not sent to the controller. In this scenario, the plant input is set to zero, the buffer is emptied, and the controller is switched off until the system state moves out of the desired region $\mathbb{B}_{d}$ and a new state measurement is received. Fig. 2 outlines the proposed algorithm. In this figure,

$$
S \triangleq\left[\begin{array}{ccccc}
0_{p} & I_{p} & 0_{p} & \ldots & 0_{p} \\
\vdots & \ddots & \ddots & \ddots & \vdots \\
0_{p} & \ldots \ldots & 0_{p} & I_{p} \\
0_{p} & \ldots \ldots \ldots & \ldots & 0_{p}
\end{array}\right] \in \mathbb{R}^{\Lambda p \times \Lambda p}, \quad b(k)=\left[\begin{array}{c}
b_{1}(k) \\
b_{2}(k) \\
\vdots \\
b_{\Lambda}(k)
\end{array}\right]
$$

where $\{b\}_{\mathbb{N}_{0}}$ denote the buffer states for a given buffer size $\Lambda \in \mathbb{N}$ and each $b_{j}(k) \in \mathbb{R}^{p}$, $j \in\{1, \ldots, \Lambda\}$.

For future use, we will denote by $N(k) \in\{0,1, \ldots, \Lambda\}$ the total number of iterations of the 
Step 1: At time $t=0$,

$$
\operatorname{SET} b(-1) \leftarrow \mathbf{0}_{\Lambda p}, k \leftarrow 0
$$

Step 2: IF $t \geq k T_{s}$,

THEN

SWITCH $\beta(k)$

CASE 2,

$$
\operatorname{SET} b(k) \leftarrow \mathbf{0}_{\Lambda p}, j \leftarrow 1 ;
$$

GOTO Step 4;

CASE 0,

SET $j \leftarrow 1, b(k) \leftarrow S b(k-1)$;

GOTO Step 4;

OTHERWISE

$$
\begin{aligned}
& \text { INPUT } x(k) ; \\
& \text { SET } \chi \leftarrow x(k), j \leftarrow 1, b(k) \leftarrow S b(k-1) ;
\end{aligned}
$$

END

END

Step 3: WHILE "sufficient processor time is available" and $j \leq \Lambda$ and time $t<(k+1) T_{s}$, EVALUATE $u_{j}(k)=\kappa(\chi)$;

IF $j=1$, THEN

$$
\begin{aligned}
& \text { OUTPUT } u_{1}(k) ; \\
& \text { SET } b(k) \leftarrow \mathbf{0}_{\Lambda p} ;
\end{aligned}
$$

END

$\operatorname{SET} b_{j}(k) \leftarrow u_{j}(k)$;

IF "sufficient processor time is not available" or $t \geq(k+1) T_{s}$, THEN GOTO Step 5;

END

$\operatorname{SET} \chi \leftarrow f\left(\chi, u_{j}(k)\right), j \leftarrow j+1$;

END

Step 4: IF $j=1$, THEN

$$
\text { OUTPUT } b_{1}(k) \text {; }
$$

END

Step 5: $\operatorname{SET} k \leftarrow k+1$ and GOTO Step 2;

Fig. 3. Algorithm $A_{1}$ 
while-loop which are carried out during the interval $t \in\left[k T_{s},(k+1) T_{s}\right)$. Thus, as described above, if $N(k) \geq 1$, then the entire sequence of tentative controls is $\left\{b_{1}(k), b_{2}(k), \ldots, b_{N(k)}(k)\right\}$ and the plant input is set to $b_{1}(k)$. If $N(k)=0$, then the plant input depends on the variable $\beta(k)$. If $\beta(k) \in\{0,1\}$ (i.e., $x(k)$ does not lie inside the desired region), then $u(k)$ is taken as the first $p$ elements of the shifted state $b(k)=S b(k-1)$. If, on the other hand, $\beta(k)=2$ indicating that $x(k) \in \mathbb{B}_{d}$, then the buffer is emptied and the plant input is set to zero, see Fig. 2.

Algorithm $\mathrm{A}_{1}$ amounts to a dynamic state feedback policy with internal state variable $b(k)$ which provides the plant input $u(k)$ and suggested plant inputs at future time steps. If new state information is received and more processor time is available, a longer trajectory of control inputs is calculated and stored in the buffer. If the buffer runs out of tentative plant inputs, then actuator values are set to zero. The algorithm does not require prior knowledge of future processor availability and hence can be employed in shared systems where the controller task can be preempted by other computational tasks at the processor.

\section{Stochastic Stability - Preliminaries}

For our subsequent analysis, it is convenient to investigate how many values in the state $b(k)$ stem from evaluating $\kappa, \ell \in \mathbb{N}_{0}$. As in [20], [22], we will refer to this value as the effective buffer length (at time $k$ ), and denote it as $\lambda(k) \in\{0,1, \ldots, \Lambda\}, k \in \mathbb{N}_{0}$ with $\lambda(-1)=0$. It is easy to see that for all $k \in \mathbb{N}_{0}$ we have

$$
\lambda(k)= \begin{cases}N(k) & \text { if } N(k) \geq 1, \\ \max \{0, \lambda(k-1)-1\}, & \text { if } N(k)=0 \text { and } \beta(k) \in\{0,1\}, \\ 0 & \text { if } \beta(k)=2 .\end{cases}
$$

To investigate stability, we make the following assumptions:

Assumption 2 (Processor availability): The sampling time of the plant (1) is such that processor availability for control at different time-instants is independent and identically distributed (i.i.d.). Thus, the process $\{N\}_{\mathbb{N}_{0}}$ has conditional probability distribution $p_{j} \triangleq \operatorname{Pr}\{N(k)=$ $j \mid \beta(k)=1\}$, where $p_{j} \in[0,1)$ are given and with $j \in\{0,1,2, \ldots, \Lambda\}$. For other realizations of $\beta(k)$, no plant inputs are calculated, thus, $\operatorname{Pr}\{N(k)=0 \mid \beta(k) \in\{0,2\}\}=1$.

Assumption 3 (Erasure channel): The binary transmission success process $\{\gamma\}_{\mathbb{N}_{0}}$ has conditional probabilities $\operatorname{Pr}\{\gamma(k)=1|| x(k) \mid \geq d\}=q, \operatorname{Pr}\{\gamma(k)=0|| x(k) \mid<d\}=1$. 
Assumption 4 (Open-loop bound): There exists $\alpha \geq \rho$ such that

$$
V\left(f\left(\chi, \mathbf{0}_{p}\right)\right) \leq \alpha V(\chi), \quad \forall \chi \in \mathbb{R}^{n} .
$$

where $\rho, V$ and $\varphi_{2}$ are as in (3). Further, $\mathbf{E}\left\{\varphi_{2}(|x(0)|)\right\}<\infty$.

It is worth noting that, by allowing for $\alpha>1$, Assumption 4 does not require that the open-loop system $x(k+1)=f\left(x(k), \mathbf{0}_{p}\right)$ be asymptotically stable. Further, note that Assumptions 1 and 4 are stated in terms of the same function $V$, see also [20, Section IV-A].

To go beyond stability and investigate stationarity, it is convenient to impose the following assumptions on the control policy $\kappa$

Assumption 5 (Continuity of $\kappa$ ): The control law $\kappa$ in (3) is such that $\kappa(x)=\mathbf{0}_{n}$ for all $x \in \mathbb{B}_{d}$ and $\kappa$ is continuous on $\mathbb{R}^{n}$.

\section{Stability with the Baseline Algorithm}

If the baseline algorithm is used and Assumption 2 holds, then

$$
x(k+1)= \begin{cases}f(x(k), \kappa(x(k))), & \text { if } N(k) \geq 1, \\ f\left(x(k), \mathbf{0}_{p}\right), & \text { if } N(k)=0 .\end{cases}
$$

The following result establishes conditions on system parameters which ensure that the closed loop (6) is stable in a stochastic sense.

Theorem 1 (Stability with baseline algorithm): Consider (6) and define $D \triangleq \varphi_{2}(d)$. Suppose that Assumptions 1 to 4 hold and that

$$
\Gamma \triangleq(1-q) \alpha+q\left(p_{0} \alpha+\left(1-p_{0}\right) \rho\right)<1,
$$

where $\rho \in[0,1)$ is the closed-loop bound in (3), $\alpha$ is the bound in (5), $q$ is the transmission success probability, and $p_{0}$ is the probability of the processor not being available for control. Then for all $x \in \mathbb{N}_{0}$,

$$
\mathbf{E}\left\{\varphi_{1}(|x(k)|)\right\} \leq \Gamma^{k} \mathbf{E}\left\{\varphi_{2}(x(0))\right\}+\frac{q\left(1-p_{0}\right)(\alpha-\rho) D}{1-\Gamma}<\infty .
$$

Proof: Note that, for i.i.d. processor and channel availabilities $\{x\}_{\mathbb{N}_{0}}$ in (6) is Markovian. This can be verified by noting that conditioning on $x(k)$ makes the event outcome $\beta(k)$ depend on $\gamma(k)$ only. To analyze stochastic stability using Lyapunov functions (see, e.g., [23]), we use the law of total expectation to write

$$
\mathbf{E}\{V(x(1)) \mid x(0)=\chi\}=\sum_{j=0}^{2} \mathbf{E}\{V(x(1)) \mid x(0)=\chi, \beta(0)=j\} \operatorname{Pr}\{\beta(0)=j \mid x(0)=\chi\} .
$$


If we now use (2), (3), (5) and the definition of $\mathbb{B}_{d}$, then:

$$
\begin{aligned}
& \mathbf{E}\{V(x(1)) \mid x(0)=\chi, \beta(0)=0\} \leq \alpha V(\chi) \\
& \mathbf{E}\{V(x(1)) \mid x(0)=\chi, \beta(0)=2\} \leq \alpha V(\chi)<\alpha \varphi_{2}(d),
\end{aligned}
$$

For $\beta(0)=1, x(0)$ is received. Using (5) and (6), we have

$$
\begin{gathered}
\mathbf{E}\{V(x(1)) \mid x(0)=\chi, \beta(0)=1\}=\sum_{j \in \mathbb{N}_{0}} \mathbf{E}\{V(x(1)) \mid x(0)=\chi, \beta(0)=1, N(0)=j\} \\
\times \operatorname{Pr}\{N(0)=j \mid x(0)=\chi, \beta(0)=1\} \leq\left(p_{0} \alpha+\left(1-p_{0}\right) \rho\right) V(\chi) .
\end{gathered}
$$

Now, if $x(0) \in \mathbb{B}_{d}$, then $\beta(0)=2$, thus $(\mathrm{V})$ and (8) provide

$$
\mathbf{E}\left\{V(x(1)) \mid x(0)=\chi \in \mathbb{B}_{d}\right\} \leq \alpha V(\chi)
$$

Further, since $\alpha-\Gamma=q\left(1-p_{0}\right)(\alpha-\rho)>0$ (see (7)) and $V(\chi)<D$ for all $\chi \in \mathbb{B}_{d}$, we have

$$
(\alpha-\Gamma) V(\chi)<(\alpha-\Gamma) D \Rightarrow \alpha V(\chi)<\Gamma V(\chi)+(\alpha-\Gamma) D, \quad \forall \chi \in \mathbb{B}_{d}
$$

On the other hand, if $x(0) \notin \mathbb{B}_{d}$, then (in view of Assumption 3), $\operatorname{Pr}\left\{\beta(0)=0 \mid x(0) \notin \mathbb{B}_{d}\right\}=$ $1-q$, and $\operatorname{Pr}\left\{\beta(0)=1 \mid x(0) \notin \mathbb{B}_{d}\right\}=q$. Thereby, substitution of (8) and (9) into (V) provides:

$$
\mathbf{E}\left\{V(x(1)) \mid x(0)=\chi \notin \mathbb{B}_{d}\right\} \leq \Gamma V(\chi) .
$$

Expressions (10)-(12) lead to:

$$
\mathbf{E}\{V(x(1)) \mid x(0)=\chi\}<\Gamma V(\chi)+(\alpha-\Gamma) D=\Gamma V(\chi)+q\left(1-p_{0}\right)(\alpha-\rho) D
$$

Consequently, Proposition 3.2 of [23], and (3) give

$$
\mathbf{E}\left\{\varphi_{1}(|x(k)|) \mid x(0)=\chi\right\} \leq \Gamma^{k} V(\chi)+\frac{q\left(1-p_{0}\right)(\alpha-\rho) D}{1-\Gamma},
$$

for all $k \in \mathbb{N}_{0}$. Using the law of total expectation and (3) yields the first inequality. The second follows from Assumption 4.

It is worth noting that whilst the condition (7) is independent of the size of $\mathbb{B}_{d}$, the ultimate bound is increasing in $d$. We can also consider two special cases. If $d=0$ and $q=1$, so that the sensor transmits at every instant $k \in \mathbb{N}_{0}$ and the communication channel does not introduce any dropouts, (7) reduces to $p_{0} \alpha+\left(1-p_{0}\right) \rho<1$, thus recovering our earlier result [20, Thm.1]. If the processor is available at every time-step (i.e., $p_{0}=0$ ), then the situation amounts to event-based 
control for non-linear systems using an erasure channel. In this case, the sufficient condition (7) becomes $(1-q) \alpha+\rho q<1$.

Theorem 2 (Stationarity with baseline algorithm): Consider (6), suppose that Assumptions 1 to 5 hold and that (7) holds. Then, there exists an invariant probability measure for $\{x\}_{\mathbb{N}_{0}}$. Furthermore, under every such invariant probability measure $\pi$,

$$
\mathbf{E}_{\pi}\left\{\varphi_{1}(|x|)\right\} \leq q\left(1-p_{0}\right)(\alpha-\rho) \varphi_{2}(d) /(1-\Gamma) .
$$

Proof: Let $\mathcal{P}\left(\mathbb{R}^{n}\right)$ denote the set of probability measures on $\mathbb{R}^{n}$ and define for every Borel $B, v_{T}(B)=(1 / T) \mathbf{E}\left\{\sum_{k=0}^{T-1} 1_{\{x(k) \in B\}}\right\}$, such that $v_{T} \in \mathcal{P}\left(\mathbb{R}^{n}\right)$ forms an expected empirical occupation measure sequence. We then have,

$$
\left\langle v_{T}, \varphi_{1}\right\rangle \triangleq \int v_{T}(d x) \varphi_{1}(|x|)=\frac{1}{T} \mathbf{E}\left\{\sum_{k=0}^{T-1} \varphi_{1}(|x(k)|)\right\} .
$$

Let $t_{0} \in \mathbb{N}$. By Theorem 1, we have that $\mathbf{E}\left\{\varphi_{1}(|x(k)|)\right\}$ and the subsequence $\left\{\left\langle v_{T}, \varphi_{1}\right\rangle, T \geq t_{0}\right\}$ are uniformly bounded by some $M_{t_{0}}<\infty$. Define $N_{r}:=\left\{x: \varphi_{1}(|x|) \leq r\right\}$. Since $\varphi_{1}$ is monotone and unbounded, by an application of Markov's inequality, we have

$$
M_{t_{0}} \geq \int v_{T}(d x) \varphi_{1}(|x|) \geq \int_{\mathbb{X} \backslash N_{r}} v_{T}(d x) \varphi_{1}(|x|) \geq r v_{T}\left(\mathbb{R}^{n} \backslash N_{r}\right) .
$$

Thus, $v_{T}\left(N_{r}\right) \geq 1-M_{t_{0}} / r$, and hence for every $\epsilon=M_{t_{0}} / r>0$, there exists a compact set $N_{M_{t_{0}} / \epsilon}=\left\{x: \varphi_{1}(|x|) \leq M_{t_{0}} / \epsilon\right\}$ such that $v_{t}\left(N_{M_{t_{0}} / \epsilon}\right) \geq 1-\epsilon$. The sequence $\left\{v_{t}, t \geq t_{0}\right\}$ is, hence, a tight sequence with a converging subsequence $v_{t_{k}}$ converging to some $v^{*} \in \mathcal{P}\left(\mathbb{R}^{n}\right)$. By (6), if $x(t) \in \mathbb{B}_{d}$ the control action is zero and outside $\mathbb{B}_{d}$, either zero control is applied or $\kappa(x(t))$ is applied. Since $\kappa$ is continuous and is zero inside $\mathbb{B}_{d}$ (see Assumption 5), the Markov chain is weak Feller. ${ }^{1}$ Consequently, it can be shown that every limit of such a subsequence is invariant (see, e.g., [24, Ch. 12]) and satisfies $\left\langle v_{T}, \varphi_{1}\right\rangle \leq M_{t_{0}}$. By Theorem 1, by increasing $t_{0}$, $M_{t_{0}}$ can be taken to be arbitrarily close to $q\left(1-p_{0}\right)(\alpha-\rho) \varphi_{2}(d) /(1-\Gamma)$.

\section{Stability With the Anytime Algorithm}

The analysis of the event-based anytime algorithm is more involved than that of the baseline system (6). First, due to buffering, $\{x\}_{\mathbb{N}_{0}}$ will in general not be a Markov process. Further, the

\footnotetext{
${ }^{1} \mathrm{~A}$ Markov chain $\{x(k)\}_{k \in \mathbb{N}_{0}}$ is (weak) Feller if $\mathbf{E}\{h(x(k+1)) \mid x(k)=\chi\}$ is continuous in $\chi$, for every continuous and bounded function $h$.
} 
distribution of $\{\beta\}_{\mathbb{N}_{0}}$ is difficult to derive for general plant models. This makes the approaches of [20], [22] insufficient to treat the present case.

For ease of exposition, we assume that the initial effective buffer length, $\lambda(0)=0$, and denote the time steps where $\lambda(k)=0$ via $\mathcal{K}=\left\{k_{i}\right\}_{i \in \mathbb{N}_{0}}$, where $k_{0}=0$ and $k_{i+1}=\inf \{k \in \mathbb{N}: k>$ $\left.k_{i}, \lambda(k)=0\right\}, i \in \mathbb{N}_{0}$. We also describe the amount of time steps between consecutive elements of $\mathcal{K}$ via the process $\left\{\Delta_{i}\right\}_{i \in \mathbb{N}_{0}}$, where $\Delta_{i} \triangleq k_{i+1}-k_{i}$. It is easy to see that

$$
\beta\left(k_{i}+\ell\right) \in\{0,1\}, \quad \forall \ell \in\left\{1,2, \ldots, \Delta_{i}-1\right\}, \quad \forall i \in \mathbb{N}_{0}
$$

whereas $\beta\left(k_{i}\right) \in\{0,1,2\}, \forall i \in \mathbb{N}_{0}$ and $x\left(k^{*}\right) \in \mathbb{B}_{d} \Rightarrow k^{*} \in \mathcal{K}$. In contrast to the cases examined in [20], [22], due to the event-triggering mechanism, $\left\{\Delta_{i}\right\}_{i \in \mathbb{N}_{0}}$ is, in general, not i.i.d. In fact, the distribution of $\Delta_{i}$ depends on $x\left(k_{i}\right)$ and is difficult to characterize. To study stability of the event-based anytime algorithm, we will develop a state-dependent random-time drift condition. Our first result, states that whilst $\{x\}_{\mathbb{N}_{0}}$ is in general not Markovian, the state sequence at the time steps $k_{i} \in \mathcal{K}$, is a Markov process.

Lemma 1 (Markov property of the sampled process): Consider (1) controlled via Algorithm $\mathrm{A}_{1}$ and suppose that Assumptions 2 and 3 hold. Then $\{x\}_{\mathcal{K}}$ is Markovian.

Proof: The definition of $\mathcal{K}$ gives that $\forall k_{i} \in \mathcal{K}$ we have $u\left(k_{i}\right)=\mathbf{0}_{p}, b\left(k_{i}\right)=\mathbf{0}_{\Lambda p}, \lambda\left(k_{i}\right)=$ $N\left(k_{i}\right)=0$. Thus, the plant state at time $k_{i+1}$ depends only on $x\left(k_{i}\right)$ and the sample paths $\left\{N\left(k_{i}+1\right), N\left(k_{i}+2\right), \ldots, N\left(k_{i+1}-1\right)\right\}$ and $\left\{\gamma\left(k_{i}+1\right), \gamma\left(k_{i}+2\right), \ldots, \gamma\left(k_{i+1}-1\right)\right\}$. The result follows since $\{N\}_{\mathbb{N}_{0}}$ and $\{\gamma\}_{\mathbb{N}_{0}}$ are i.i.d.

The following result provides a sufficient condition for stochastic stability of the closed loop when the event-based anytime control algorithm of Section III is used over an erasure channel.

Theorem 3 (Stability with Algorithm $A_{1}$ ): Suppose that Assumptions 1 to 4 hold and define

$$
\Omega \triangleq \alpha \sum_{j \in \mathbb{N}} \rho^{j-1} \operatorname{Pr}\left\{\Delta_{i}=j \mid \beta\left(k_{i+1}\right) \neq 2\right\} .
$$

If Algorithm $\mathrm{A}_{1}$ is used and $\Omega<1$, then

$$
\max _{k \in\left\{k_{i}, k_{i}+1, \ldots, k_{i+1}-1\right\}} \mathbf{E}\left\{\varphi_{1}(|x(k)|)\right\} \leq \frac{1+\alpha-\rho}{1-\rho} \Omega^{i} \mathbf{E}\left\{\varphi_{2}(x(0))\right\}+\frac{\varphi_{2}(d)}{1-\Omega}<\infty, \quad \forall i \in \mathbb{N} .
$$

Proof: We first note that for all $k_{i} \in \mathcal{K}$ and $\ell \in\left\{1, \ldots, \Delta_{i}-1\right\}, u\left(k_{i}\right)=\mathbf{0}_{p}$ and $u\left(k_{i}+\ell\right)=$ $\kappa\left(x\left(k_{i}+\ell\right)\right)$. Therefore, the function $V\left(x\left(k_{i+1}\right)\right)$ can be bounded by using (3) and (5), leading to

$$
\mathbf{E}\left\{V\left(x\left(k_{i+1}\right)\right) \mid x\left(k_{i}\right)=\chi, \Delta_{i}=j\right\} \leq \alpha \rho^{j-1} V(\chi), \forall \chi \in \mathbb{R}^{n} .
$$


To account for event-based transmission, we consider instances where the buffer is emptied triggered by $\beta(k)=2$. At these instances, (16) holds; further, $V\left(k_{i+1}\right)<D \triangleq \varphi_{2}(d)$. Thus,

$$
\mathbf{E}\left\{V\left(x\left(k_{i+1}\right)\right) \mid x\left(k_{i}\right)=\chi, \Delta_{i}=j, \beta\left(k_{i+1}\right)=2\right\}<D, \quad \forall j \in \mathbb{N} .
$$

By using the law of total expectation twice, we thus obtain,

$$
\begin{aligned}
& \mathbf{E}\left\{V\left(x\left(k_{i+1}\right)\right) \mid x\left(k_{i}\right)=\chi\right\}=\mathbf{E}\left\{V\left(x\left(k_{i+1}\right)\right) \mid x\left(k_{i}\right)=\chi, \beta\left(k_{i+1}\right)=2\right\} \operatorname{Pr}\left\{\beta\left(k_{i+1}\right)=2 \mid x\left(k_{i}\right)=\chi\right\} \\
& +\mathbf{E}\left\{V\left(x\left(k_{i+1}\right)\right) \mid x\left(k_{i}\right)=\chi, \beta\left(k_{i+1}\right) \neq 2\right\} \operatorname{Pr}\left\{\beta\left(k_{i+1}\right) \neq 2 \mid x\left(k_{i}\right)=\chi\right\} \\
& \leq D+\mathbf{E}\left\{V\left(x\left(k_{i+1}\right)\right) \mid x\left(k_{i}\right)=\chi, \beta\left(k_{i+1}\right) \neq 2\right\} \\
& =D+\sum_{j \in \mathbb{N}} \mathbf{E}\left\{V\left(x\left(k_{i+1}\right)\right) \mid x\left(k_{i}\right)=\chi, \beta\left(k_{i+1}\right) \neq 2, \Delta_{i}=j\right\} \operatorname{Pr}\left\{\Delta_{i}=j \mid x\left(k_{i}\right)=\chi, \beta\left(k_{i+1}\right) \neq 2\right\} \\
& \leq D+\sum_{j \in \mathbb{N}} \alpha \rho^{j-1} V(\chi) \operatorname{Pr}\left\{\Delta_{i}=j \mid x\left(k_{i}\right)=\chi, \beta\left(k_{i+1}\right) \neq 2\right\}=D+\Omega V(\chi), \forall \chi \in \mathbb{R}^{n}
\end{aligned}
$$

with $\Omega$ as in (14) and where, to derive the last equality, we have used Assumption 2. Since $\{x\}_{\mathcal{K}}$ is Markovian, [23, Prop. 3.2] yields that $\Omega<1$ guarantees

$$
\mathbf{E}\left\{V\left(x\left(k_{i}\right)\right) \mid x\left(k_{0}\right)=\chi\right\} \leq \Omega^{i} V(\chi)+\frac{D}{1-\Omega}, \quad \forall i \in \mathbb{N}_{0} .
$$

Now, since (16) holds, by a method similar to the one used in the proof of [22, Thm.1], we can establish the (admittedly loose) bound:

$$
\mathbf{E}\left\{\sum_{k=k_{i}}^{k_{i+1}-1} V(x(k)) \mid x\left(k_{0}\right)=\chi\right\} \leq \frac{1+\alpha-\rho}{1-\rho} \Omega^{i} V(\chi)+\frac{D}{1-\Omega}, \quad \forall i \in \mathbb{N} .
$$

Using the law total expectation, (3) and Assumption 4 gives (15).

The above result establishes a sufficient condition for the system to be stochastically stable. The quantity (14) is stated in terms of a conditional distribution of $\Delta_{i}$, which can be characterized as follows:

Lemma 2 (Conditional distribution of $\Delta_{i}$ ): Suppose that Assumptions 2 and 3 hold and that Algorithm $\mathrm{A}_{1}$ is used. We then have

$$
\frac{\operatorname{Pr}\left\{\Delta_{i}=j \mid \beta\left(k_{i+1}\right) \neq 2\right\}}{1-q+p_{0} q}=\left\{\begin{array}{ll}
1 & \text { if } j=1, \\
\theta^{T} G^{j-2} e_{1} & \text { if } j \geq 2,
\end{array} \quad \forall(i, j) \in \mathbb{N}_{0} \times \mathbb{N}\right.
$$

where $\theta^{T}=q\left[\begin{array}{lll}p_{1} & \ldots & p_{\Lambda}\end{array}\right]$ and $e_{1}^{T}=\left[\begin{array}{llll}1 & 0 & \ldots & 0\end{array}\right]$. In (20), the entries of the matrix $G=\left[g_{\ell j}\right], \ell, j \in\{1,2, \ldots, \Lambda\}$ are $g_{\ell j}=p_{j} q, \forall(\ell, j) \in\{3,4, \ldots, \Lambda\} \times\{1,2, \ldots, \ell-2\} \cup$ $\{1,2, \ldots, \Lambda\} \times\{\ell, \ell+1, \ldots, \Lambda\} ;$ and $g_{\ell(\ell-1)}=1-q+\left(p_{0}+p_{\ell-1}\right) q, \forall \ell \in\{2,3, \ldots, \Lambda\}$. 
Proof: We first note that our focus is on the time sequences of the form $\mathcal{I}_{i} \triangleq\left\{k_{i}+\right.$ $\left.1, \ldots, k_{i+1}\right\}$ where $k_{i} \in \mathcal{K}, i \in \mathbb{N}_{0}$ and where $\beta(k) \neq 2, \forall k \in \mathcal{I}_{i}$. Given Assumptions 2 and 3 and the buffering mechanism described in Section III, it follows that $\{\lambda(k)\}$ during every interval $k \in \mathcal{I}_{i}, i \in \mathbb{N}_{0}$, is a homogeneous Markov Chain. The process $\Delta_{i}$ then amounts to the first return times to 0 of this finite Markov Chain. To characterize the latter, we need to evaluate the transition probabilities $g_{\ell j} \triangleq \operatorname{Pr}\left\{\lambda(k+1)=j \mid \lambda(k)=\ell, k \in \mathcal{I}_{i}, k+1 \in \mathcal{I}_{i}\right\}$. Without loss of generality, we will set $k=0$. We begin by considering transitions from $\ell \in\{0,1\}$ to 0 :

$$
\begin{aligned}
g_{\ell 0}= & \operatorname{Pr}\{N(1)=0 \mid \beta(1)=0\} \operatorname{Pr}\{\beta(1)=0 \mid \beta(1) \neq 2\} \\
& +\operatorname{Pr}\{N(1)=0 \mid \beta(1)=1\} \operatorname{Pr}\{\beta(1)=1 \mid \beta(1) \neq 2\}=(1-q)+p_{0} q, \quad \forall \ell \in\{0,1\} .
\end{aligned}
$$

For $\ell \in\{2,3, \ldots, \Lambda\}$, we have $g_{\ell 0}=0$. The buffer length diminishes by one for the scenarios considered below:

$$
\begin{aligned}
g_{\ell(\ell-1)}= & \operatorname{Pr}\{N(1)=0 \mid \beta(1)=0\} \operatorname{Pr}\{\beta(1)=0 \mid \beta(1) \neq 2\}+\operatorname{Pr}\{N(1)=0 \mid \beta(1)=1\} \\
& \times \operatorname{Pr}\{\beta(1)=1 \mid \beta(1) \neq 2\}+\operatorname{Pr}\{N(1)=\ell-1 \mid \beta(1)=1\} \operatorname{Pr}\{\beta(1)=1 \mid \beta(1) \neq 2\} \\
= & (1-q)+p_{0} q+p_{\ell-1} q, \quad \forall \ell \in\{2,3, \ldots, \Lambda\} .
\end{aligned}
$$

The other transitions are related to when $\lambda(k+1)=N(k+1)$, for $(\ell, j) \in\{\{3,4, \ldots, \Lambda\} \times$ $\{1,2, \ldots, \ell-2\}\} \cup\{\{1,2, \ldots, \Lambda\} \times\{\ell, \ell+1, \ldots, \Lambda\}\} \cup\{0 \times\{1,2, \ldots, \Lambda\}\}$. Here we have:

$$
\begin{aligned}
g_{\ell j}= & \operatorname{Pr}\{\lambda(1)=j \mid \lambda(0)=\ell, \beta(1)=0\} \operatorname{Pr}\{\beta(1)=0 \mid \beta(1) \neq 2\} \\
& +\operatorname{Pr}\{\lambda(1)=j \mid \lambda(0)=\ell, \beta(1)=1\} \operatorname{Pr}\{\beta(1)=1 \mid \beta(1) \neq 2\} \\
= & \operatorname{Pr}\{N(1)=j \mid \beta(1)=0\} \operatorname{Pr}\{\beta(1)=0 \mid \beta(1) \neq 2\}+\operatorname{Pr}\{N(1)=j \mid \beta(1)=1\} \\
& \times \operatorname{Pr}\{\beta(1)=1 \mid \beta(1) \neq 2\}=0(1-q)+p_{j} q=p_{j} q .
\end{aligned}
$$

The derivation of (20) now follows as in [22, Lemma 2] by setting up a recursion on the first passage time of state $\ell \in\{1, \ldots, \Lambda\}$ to 0 and then considering the transitions away from 0 .

As a consequence of Lemma $2, \Omega$ in (14) can be written as:

$$
\Omega=\alpha\left(1-q+p_{0} q\right)\left(1+\rho \theta^{T}(I-\rho G)^{-1} e_{1}\right)
$$

and the stability condition in Theorem $3, \Omega<1$, becomes

$$
\left[\begin{array}{lll}
p_{1} & \ldots & p_{\Lambda}
\end{array}\right]\left(I_{\Lambda}-\rho G\right)^{-1} e_{1}<\frac{1-\alpha+\alpha q\left(1-p_{0}\right)}{\alpha \rho q\left(1-q\left(1-p_{0}\right)\right)},
$$

which is independent of the size of $\mathbb{B}_{d}$. 
Sufficient conditions for stationarity can be stated as follows:

Theorem 4 (Stationarity with Algorithm $A_{1}$ ): Suppose that Assumptions 1 to 5 hold. If Algorithm $\mathrm{A}_{1}$ is used and $\Omega<1$, then there exists an invariant probability measure for $\{x\}_{\mathcal{K}}$ as well as for the aggregated Markov process, $\left\{x_{[k, k-(\Lambda-1)]}\right\}_{k \in \mathbb{N}}$, where

$$
x_{[k, k-(\Lambda-1)]} \triangleq\{x(k), x(k-1), \cdots, x(k-\Lambda+1)\} .
$$

Furthermore, under every invariant probability measure $\pi, \mathbf{E}_{\pi}\{V(x)\}<\varphi_{2}(d) /(1-\Omega)$.

Proof: First note that if $N(k) \geq 1$, then $u(k)$ is determined by the current state. If the processor is not available, then either $u(k)$ has been determined by the states which are at most $\Lambda$ time stages old, or $u(k)=\mathbf{0}_{p}$. Since the processor availability is independent of the state, the stochastic process $\left\{x_{[k, k-\Lambda+1]}\right\}$ is Markovian. Let $\mathbf{z}(k) \triangleq x_{[k, k-\Lambda+1]}$. From Assumption 5, $\{\mathbf{z}\}_{\mathbb{N}_{0}}$ is also weak Feller.

We first invoke Theorem 2.1 in [25] with $\mathcal{K}$ containing the sequence of stopping times. Since

$$
\mathbf{E}\left\{V\left(x\left(k_{i+1}\right)\right) \mid x\left(k_{i}\right)=\chi\right\} \leq V(\chi)-(1-\Omega) V(\chi)+D, \quad \forall \chi \in \mathbb{R}^{n},
$$

and the sampled chain is weak Feller, it follows that $\{x\}_{\mathcal{K}}$ admits an invariant probability measure.

Define $\tilde{V}(\mathbf{z}(k)) \triangleq V(x(k))$. Now, note that by (18), with $\Omega<1, \mathbf{E}\left\{\tilde{V}\left(\mathbf{z}\left(k_{i+1}\right)\right) \mid \mathbf{z}\left(k_{i}\right)=\right.$ $\chi\} \leq D+\Omega \tilde{V}(\chi), \forall \chi$. Thus, $\mathbf{E}\left\{\tilde{V}\left(\mathbf{z}\left(k_{i+1}\right)\right) \mid \mathbf{z}\left(k_{i}\right)=\chi\right\} \leq \tilde{V}(\chi)-(1-\Omega) \tilde{V}(\chi)+D, \forall \chi$, and since $V$ is monotone increasing and by Assumption 4, there exists a compact set $\mathcal{S}$ such that for $1-\Omega>\zeta>0, \mathbf{E}\left\{V\left(x\left(k_{i+1}\right)\right) \mid x\left(k_{i}\right)=x\right\} \leq V(x)-\zeta V(x)+D 1_{x \in \mathcal{S}}, \quad \forall x \in \mathbb{R}^{n}$. Since $V(x(t))$ is bounded from below outside $\mathbb{B}_{d}$, and $x(k) \notin \mathbb{B}_{d}$ for $k \notin \mathcal{K}$, and that (19) implies that for some $M_{1}<\infty$

$$
\mathbf{E}\left\{\sum_{k=k_{i}}^{k_{i+1}-1} V(x(k)) \mid x\left(k_{0}\right)=\chi\right\} \leq M_{1}
$$

it follows that $\sup _{x_{k_{i}}} \mathbf{E}\left\{k_{i+1}-k_{i} \mid x_{k_{i}}\right\}<\infty$. Finally, by Assumption 4, if $x_{t} \in \mathcal{S}$ then $x_{[t+\Lambda-1, t]} \in$ $\overline{\mathcal{S}}$ where $\overline{\mathcal{S}}$ is a compact set. Thus, Theorem 2.2 in [25] implies that there exists an invariant probability distribution, $\pi$, for $\{\mathbf{z}\}_{\mathbb{N}_{0}}$.

Since (21) holds, with $P^{m} V(\chi):=\mathbf{E}\left\{V\left(x\left(k_{m}\right)\right) \mid x\left(k_{0}\right)=\chi\right\}$, following arguments similar to the proof of Theorem 2.2 of [25], for every realization of $x\left(k_{0}\right)$, it follows that

$$
(1-\Omega) \limsup _{T \rightarrow \infty} \frac{1}{T} \mathbf{E}\left\{\sum_{i=0}^{T-1} V\left(x\left(k_{i}\right)\right)\right\} \leq \limsup _{T \rightarrow \infty} \frac{1}{T}\left(V\left(x\left(k_{0}\right)\right)+\sum_{i=0}^{T-1} D\right) .
$$




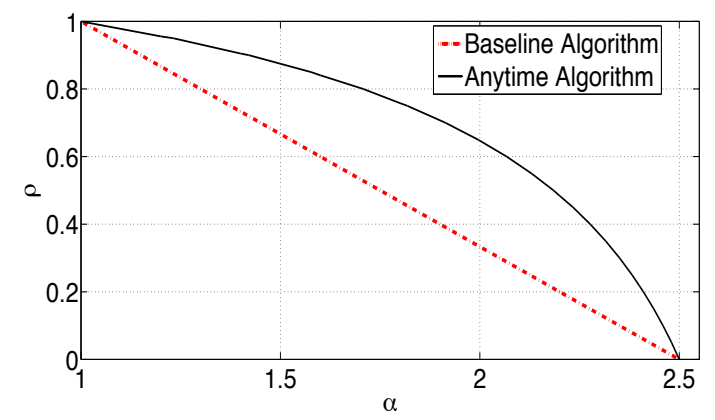

Fig. 4. Boundaries of stability: $\Omega=1$ (solid line) and $\Gamma=1$ (dashed).

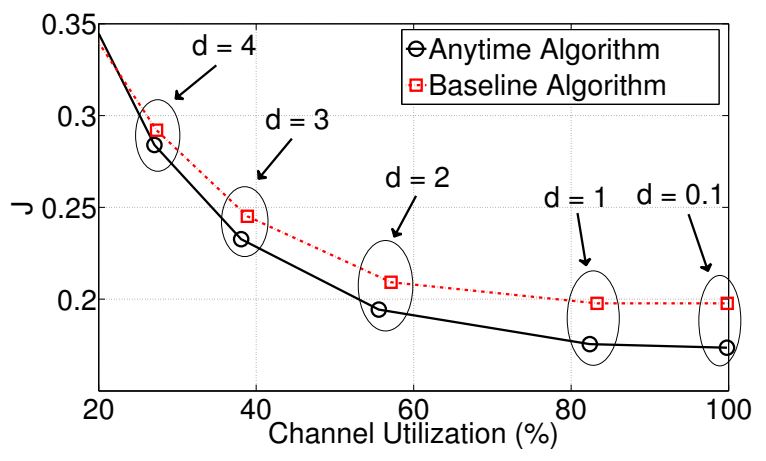

Fig. 5. Empirical cost versus channel utilization for different values of $d$.

Thus, $\lim \sup _{T \rightarrow \infty}(1 / T) \sum_{m=0}^{T-1} P^{m} V\left(x\left(k_{m}\right)\right) \leq D /(1-\Omega)$. Applying Fatou's lemma, we obtain $\limsup _{T \rightarrow \infty} \mathbf{E}_{\pi}\left\{\frac{1}{T} \sum_{i=0}^{T-1} \min \left(N, V\left(x\left(k_{i}\right)\right)\right)\right\} \leq \mathbf{E}_{\pi}\left\{\limsup _{T \rightarrow \infty} \frac{1}{T} \sum_{i=0}^{T-1} \min \left(N, V\left(x\left(k_{i}\right)\right)\right)\right\} \leq \frac{D}{1-\Omega}$.

Then, by the monotone convergence theorem, by letting $N \rightarrow \infty$,

$$
\limsup _{T \rightarrow \infty} \mathbf{E}_{\pi}\left\{\frac{1}{T} \sum_{i=0}^{T-1} V\left(x\left(k_{i}\right)\right)\right\} \leq \frac{D}{1-\Omega} .
$$

Thus, there exists an invariant probability measure both for the original chain and for the sampled chain; under every such invariant probability measure $\pi, \mathbf{E}_{\pi}\{V(x)\}<D /(1-\Omega)$.

\section{NUMERICAL EXAMPLES}

We first compare the stability conditions derived for a specific case. Suppose that the buffer length is given by $\Lambda=4$, whereas $p_{i}=0.2, i \in\{0, \ldots, 4\}$, and $q=0.75$. The stability region boundaries, see (7) and (14), in terms of $\alpha$ and $\rho$ are depicted in Fig. 4. It can be seen that 
the guaranteed stable region (under the curve) provided by our results is larger when using Algorithm $\mathrm{A}_{1}$ than when using (4).

Next, we consider an open-loop unstable constrained plant model of the form (1), but with additive noise:

$$
\left[\begin{array}{c}
x_{1}(k+1) \\
x_{2}(k+1)
\end{array}\right]=\left[\begin{array}{c}
x_{2}(k)+u_{1}(k) \\
-\operatorname{sat}\left(x_{1}(k)+x_{2}(k)\right)+u_{2}(k)
\end{array}\right]+\left[\begin{array}{c}
w_{1}(k) \\
w_{2}(k)
\end{array}\right]
$$

where

$$
\operatorname{sat}(\mu)= \begin{cases}-10, & \text { if } \mu<-10, \\ \mu & \text { if } \mu \in[-10,10], \\ 10, & \text { if } \mu>10,\end{cases}
$$

see [20, Example 2]. The initial condition $x(0)$ and the disturbance $w(k)$ are zero-mean i.i.d.

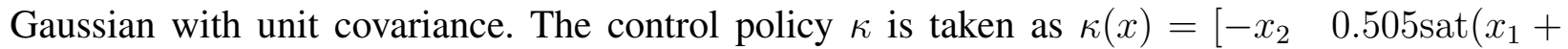
$\left.\left.x_{2}\right)\right]^{T}, x \in \mathbb{R}^{2}$. If we choose $V(x)=2|x|$, then direct calculations give that

$$
\begin{aligned}
& V(f(x, \kappa(x)))=0.99\left|\operatorname{sat}\left(x_{1}+x_{2}\right)\right| \leq 0.99\left|x_{1}+x_{2}\right| \\
& \quad \leq 1.98 \max \left\{\left|x_{1}\right|,\left|x_{2}\right|\right\}-\max \left\{\left|x_{1}\right|,\left|x_{2}\right|\right\}+|x| \leq 1.98|x| .
\end{aligned}
$$

Thus, Assumption 1 holds with $\rho=0.99$, and $\varphi_{1}(s)=\varphi_{2}(s)=2 s$. Processor availability and $\Lambda$ are taken as above, but we now set $q=0.4$. Performance is evaluated through the empirical cost $J \triangleq \frac{1}{50}\left(\sum_{k=0}^{49}|x(k)|^{2}\right)$ and the Channel Utilization (\%), calculated as

$\frac{\text { Total number of time steps at which } \beta(k) \neq 2}{\text { Total number of time steps }}(\%)$.

By averaging over $10^{4}$ realizations, Fig. 5 is obtained. As can be seen in that figure, the proposed event-based anytime control algorithm gives better trade-offs between empirical cost and channel utilization.

\section{CONCLUSIONS}

This work considered the control of a non-linear process with both communication and processing constraints. A sensor node transmits data to the controller across a channel that stochastically erases data. The control algorithm is executed over a processor that can provide only limited, time-varying and a priori unknown processing resources. To reduce the communication frequency, the sensor utilizes an event-triggered scheme. Similarly, to better utilize the processor availability, 
the control input is calculated by using an anytime control algorithm. For the resulting system, we present stochastic stability and stationarity results. Numerical studies illustrate that significant performance gains can be obtained by using the proposed algorithm. Future work includes the extension of the analysis to noisy systems, and establishing further stability properties such as ergodicity and rates of convergence to equilibrium.

\section{REFERENCES}

[1] V. Gupta, A. F. Dana, J. P. Hespanha, R. M. Murray, and B. Hassibi, "Data transmission over networks for estimation and control," IEEE Trans. Automat. Contr., vol. 54, pp. 1807-1819, Aug. 2009.

[2] O. C. Imer, S. Yüksel, and T. Bassar, "Optimal control of LTI systems over unreliable communication links," Automatica, vol. 42, pp. 1429-1439, Sept. 2006.

[3] L. Schenato, B. Sinopoli, M. Franceschetti, K. Poolla, and S. S. Sastry, "Foundations of control and estimation over lossy networks," Proc. IEEE, vol. 95, pp. 163-187, Jan. 2007.

[4] D. E. Quevedo and D. Nešić, "Robust stability of packetized predictive control of nonlinear systems with disturbances and Markovian packet losses," Automatica, vol. 48, pp. 1803-1811, Aug. 2012.

[5] L. Li, M. Lemmon, and X. Wang, "Event-triggered state estimation in vector linear processes," in Proc. Amer. Contr. Conf., pp. 2138-2143, 2010.

[6] P. Tabuada, "Event-triggered real-time scheduling of stabilizing control tasks," IEEE Trans. Automat. Contr., vol. 52, pp. 1680-1685, Sept. 2007.

[7] Y. Xu and J. Hespanha, "Optimal communication logics in networked control systems," in Proc. IEEE Conf. Decis. Contr., pp. 3527-3532, 2004.

[8] C. Ramesh, H. Sandberg, and K. H. Johansson, "Steady state performance analysis of multiple state-based schedulers with CSMA," in Proc. IEEE Conf. Decis. Contr., 2011.

[9] M. Xia, V. Gupta, and P. J. Antsaklis, "Networked state estimation over a shared communication medium," in Proc. Amer. Contr. Conf., 2013.

[10] M. Rabi and K. H. Johansson, "Scheduling packets for event-triggered control," in Proc. Europ. Contr. Conf., pp. 37793784, 2009.

[11] R. Blind and F. Allgöwer, "Analysis of networked event-based control with a shared communication medium: Part 1 pure aloha," in Proc. IFAC World Congr., 2011.

[12] A. Cervin and T. Henningsson, "Scheduling of event-triggered controllers on a shared network," in Proc. IEEE Conf. Decis. Contr., pp. 3601-3606, 2008.

[13] L. K. McGovern and E. Feron, "Closed-loop stability of systems driven by real-time dynamic optimization algorithms," in Proc. IEEE Conf. Decis. Contr., vol. 4, (Phoenix, AZ), pp. 3690-3696, Dec. 1999.

[14] D. Henriksson and J. Åkesson, "Flexible implementation of model predictive control using sub-optimal solutions," Tech. Rep. Internal Report No. TFRT-7610-SE, Dep. of Automatic Control, Lund University, 2004.

[15] P. Andrianiaina, A. Seuret, and D. Simon, "Robust system control method with short execution deadlines." European Patent Application EP 2568346 A1, Airbus Operations Toulouse, March 2013.

[16] A. Cervin, M. Velasco, P. Martí, and A. Camacho, "Optimal online sampling period assignment: Theory and experiments," IEEE Trans. Contr. Syst. Technol., vol. 18, June 2010.

[17] R. Bhattacharya and G. J. Balas, "Anytime control algorithms: Model reduction approach," AIAA Journal of Guidance, Control and Dynamics, vol. 27, pp. 767-776, Sept.-Oct. 2004.

[18] L. Greco, D. Fontanelli, and A. Bicchi, "Almost sure stability of anytime controllers via stochastic scheduling," in Proc. IEEE Conf. Decis. Contr., (New Orleans, LA), pp. 5640-5645, Dec. 2007. 
[19] V. Gupta and F. Luo, "On a control algorithm for time-varying processor availability," IEEE Trans. Automat. Contr., vol. 58, Mar. 2013.

[20] D. E. Quevedo and V. Gupta, "Sequence-based anytime control," IEEE Trans. Automat. Contr., vol. 58, pp. 377-390, Feb. 2013.

[21] C. Ramesh, H. Sandberg, and K. H. Johansson, "On the dual effect in state-based scheduling of networked control systems," in Proc. Amer. Contr. Conf., pp. 2216-2221, 2011.

[22] D. E. Quevedo and V. Gupta, "Stability of sequence-based anytime control with Markovian processor availability," in Proc. Austr. Contr. Conf., 2011.

[23] S. P. Meyn, "Ergodic theorems for discrete time stochastic systems using a stochastic Lyapunov function," SIAM Journal on Control and Optimization, vol. 27, pp. 1409-1439, Nov. 1989.

[24] S. Meyn and R. L. Tweedie, Markov Chains and Stochastic Stability. Cambridge University Press, 2009.

[25] S. Yüksel and S. P. Meyn, "Random-time, state-dependent stochastic drift for Markov chains and application to stochastic stabilization over erasure channels," IEEE Trans. Automat. Contr., vol. 58, no. 1, pp. 47-59, 2013. 


\title{
Stochastic Stability of Event-triggered Anytime Control
}

\author{
Daniel E. Quevedo, Member, IEEE, Vijay Gupta, Member, IEEE, Wann-Jiun \\ Ma, Student Member, IEEE, Serdar Yüksel, Member, IEEE
}

\begin{abstract}
We investigate control of a non-linear process when communication and processing capabilities are limited. The sensor communicates with a controller node through an erasure channel which introduces i.i.d. packet dropouts. Processor availability for control is random and, at times, insufficient to calculate plant inputs. To make efficient use of communication and processing resources, the sensor only transmits when the plant state lies outside a bounded target set. Control calculations are triggered by the received data. If a plant state measurement is successfully received and while the processor is available for control, the algorithm recursively calculates a sequence of tentative plant inputs, which are stored in a buffer for potential future use. This safeguards for time-steps when the processor is unavailable for control. We derive sufficient conditions on system parameters for stochastic stability of the closed loop and illustrate performance gains through numerical studies.
\end{abstract}

\section{INTRODUCTION}

Due to the tight coupling among the cyber and the physical cores in many cyber-physical systems, it is imperative to develop systematic design principles for controllers with limited communication and processing resources. Both the areas of control with communication constraints

D. Quevedo is with the School of Electrical Engineering \& Computer Science, The University of Newcastle, Australia, dquevedo@ieee.org. V. Gupta and W. J. Ma are with the Department of Electrical Engineering, University of Notre Dame, USA, vgupta2@nd.edu, wma1@nd.edu. S. Yüksel is with the Department of Mathematics and Statistics, Queen's University, Kingston, ON K7L 3N6 Canada, yuksel@mast.queensu.ca. Research supported for the first author under Australian Research Council's Discovery Projects funding scheme (project number DP0988601) and in part for the second and third authors by NSF awards 0846631 and 0834771. 


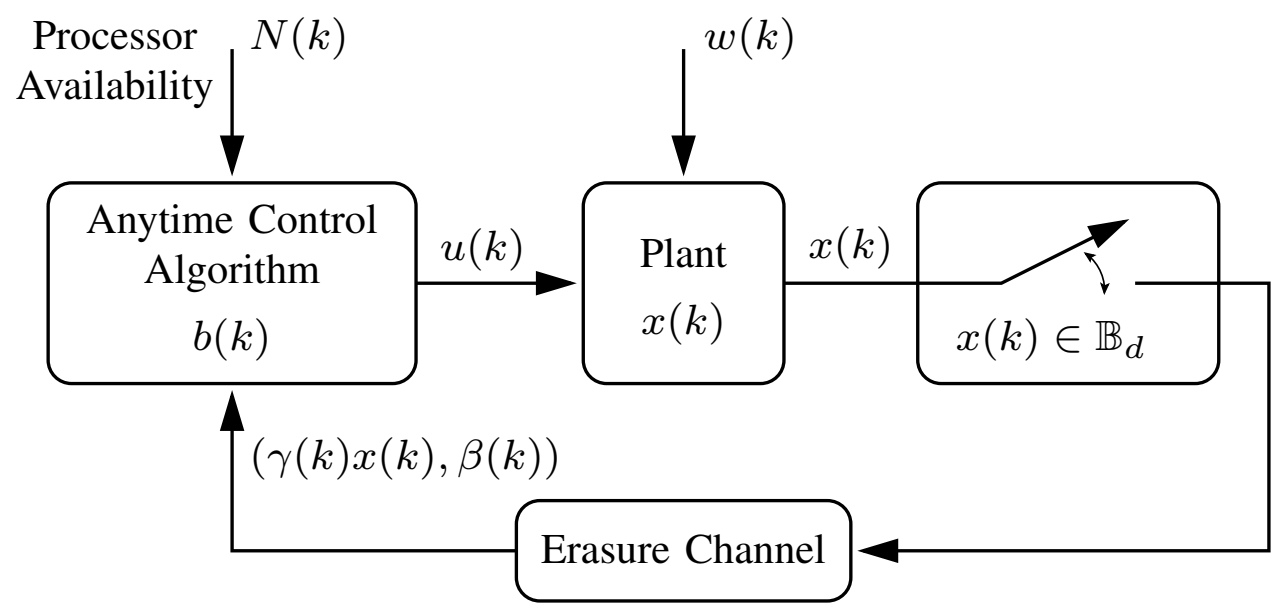

Fig. 1. Event-triggered Anytime Control with Unreliable Communications.

and control with limited and time-varying processor availability have accordingly received much attention.

Control design in the presence of practical communication channels and protocols has been studied in the area of networked control systems. Of particular interest to the present work is the literature on control across analog erasure channels; see, e.g., [1]-[4]. Apart from arising from data transmission across a wireless channel, data loss may also arise due to congestion in a communication network, possibly transmitted by a control loop. To minimize this source of data loss, one can conceive event triggered communication schemes in which sensors transmit information only if the system state exceeds a certain bound; see, e.g., [5]-[9]. Recently, work has also been done on designing event triggering rules to ensure stability in the face of data dropouts. However, most works are restricted to single integrator dynamics [10], [11] or are numerical studies [12].

On the other hand, various works have also considered the impact of limited or time-varying processing power on closed-loop control [13]-[15]. Interestingly, event-triggered and self-triggered updates of the control inputs have also been proposed to ensure less demand on the processor on average by calculating the control input on demand [6], [16]. The direction of anytime control has also shown promise [17]-[20]. Such algorithms calculate a coarse control input even with limited processing resources and refine the input as more processing resources become available. 
The quality of control inputs is thus time-varying, but no control input is obtained only rarely.

Notwithstanding the advances discussed above, relatively few works have considered control design under both limited communication and processing resources. Optimal control design for arbitrary non-linear processes under communication and processing constraints is likely a challenging problem, since certainty equivalence would not hold in general [21]. Accordingly, in the present note we consider a pre-designed control law, and focus on the implementation of this controller in the presence of both communication and processing limitations. As depicted in Fig. 1, we consider a discrete-time non-linear plant being controlled across a communication network that stochastically erases data transmitted across it. To reduce congestion in the network, the sensor employs an event triggered communication strategy. However, due to time-varying availability of the processing resources, it is not guaranteed that the processor can calculate a control input at all time steps when the sensor transmits (even if the network does not erase the data). To maximally utilize the processing resources, the controller employs an anytime control algorithm. Under such a setting, we analyze stochastic stability of the closed loop. Our main stability results are stated in terms of an inequality that relates open-loop growth of the plant state, packet erasure probability, and parameters of the processor availability model. For the particular case where processing resources are available at every time step, our result reduces to a sufficient condition for stochastic stability of non-linear control in where sensor communicates according to an event-triggering condition across an analog i.i.d. erasure link. This may be of independent interest.

Notation: We write $\mathbb{N}$ for $\{1,2, \ldots\}$ and $\mathbb{N}_{0}$ for $\mathbb{N} \cup\{0\}$. $\mathbb{R}$ represents the real numbers and $\mathbb{R}_{\geq 0} \triangleq[0, \infty)$. The $p \times p$ identity matrix is denoted via $I_{p}, 0_{p \times q}$ is the $p \times q$ all-zeroes matrix, $0_{p} \triangleq 0_{p \times p}$, and $\mathbf{0}_{p} \triangleq 0_{p \times 1}$. The notation $\{x\}_{\mathcal{K}}$ stands for $\{x(k): k \in \mathcal{K}\}$, where $\mathcal{K} \subseteq \mathbb{N}_{0}$. We adopt the conventions $\sum_{k=\ell_{1}}^{\ell_{2}} a_{k}=0$ and $\prod_{k=\ell_{1}}^{\ell_{2}} a_{k}=1$, if $\ell_{1}>\ell_{2}$ and irrespective of $a_{k} \in \mathbb{R}$. The superscript ${ }^{T}$ refers to transpose. The Euclidean norm of a vector $x$ is denoted via $|x|=\sqrt{x^{T} x}$. A function $\varphi: \mathbb{R}_{\geq 0} \rightarrow \mathbb{R}_{\geq 0}$ is of class- $\mathscr{K}_{\infty}\left(\varphi \in \mathscr{K}_{\infty}\right)$, if it is continuous, zero at zero, strictly increasing, and unbounded. The probability of an event $\Omega$ is denoted by $\operatorname{Pr}\{\Omega\}$ and the conditional probability of $\Omega$ given $\Gamma$ by $\operatorname{Pr}\{\Omega \mid \Gamma\}$. The expected value of a random variable $x$ given $\Gamma$ is denoted by $\mathbf{E}\{x \mid \Gamma\}$, while $\mathbf{E}\{x\}$ refers to the unconditional expectation. The expression $x \sim \nu$ denotes that the random variable $x$ has probability distribution $\nu$ and $\mathbf{E}_{\nu}\{x\}$ denotes the expectation under probability distribution $\nu$. 


\section{EVEnT-DRIVEn CONTROL OVER AN ERASURE CHANNEL}

We consider non-linear (and possibly open-loop unstable) plants, sampled periodically with sampling interval $T_{s}>0$ (see Fig. 1),

$$
x(k+1)=f(x(k), u(k)), \quad k \in \mathbb{N}_{0},
$$

where $x \in \mathbb{R}^{n}$ is the plant state, and $u \in \mathbb{U} \subseteq \mathbb{R}^{p}$ with $\mathbf{0}_{p} \in \mathbb{U}$ is the (possibly constrained) plant input. The initial state $x(0)$ is arbitrarily distributed. The plant is equipped with a sensor, which has direct access to the plant state at the sampling instants $k \in \mathbb{N}_{0}$.

To save on communication expenditure, the sensor adopts an event-triggered transmission strategy, in which the sensor transmits only at instances $k \in \mathbb{N}_{0}$, where $x(k) \notin \mathbb{B}_{d} \triangleq\{x \in$ $\left.\mathbb{R}^{n}:|x|<d\right\}$. This transmission is across an erasure channel which introduces random packet dropouts. To keep communication costs low, the controller does not send acknowledgments back to the sensor and no re-transmissions are allowed. We introduce two discrete random processes, namely $\{\gamma\}_{\mathbb{N}_{0}}$ and $\{\beta\}_{\mathbb{N}_{0}}$. The binary transmission success process $\{\gamma\}_{\mathbb{N}_{0}}$ describes packet loss: a successful transmission at time $k$ is denoted by $\gamma(k)=1$ and a packet erasure by $\gamma(k)=0$. The ternary process $\{\beta\}_{\mathbb{N}_{0}}$ incorporates the event-based transmission rule,

$$
\beta(k)= \begin{cases}\gamma(k) & \text { if the sensor transmitted at time } k, \\ 2 & \text { if the sensor did not transmit at time } k .\end{cases}
$$

Thus, $\beta(k)=2 \Leftrightarrow|x(k)|<d$. We assume that $\beta(k)$ is known to the controller at time $k$ through monitoring of received energy in the sensor transmission band. Transmission outcomes trigger the functions carried out by the controller. The scalar $d \in \mathbb{R}_{\geq 0}$ is a design parameter, which determines communication channel utilization and control performance. Elucidating the trade-off between these quantities is one of the motivations of the present work.

When implementing discrete-time control systems, it is generally assumed that the processing resources available to the controller are such that the desired control law can be evaluated within a fixed time-delay, say $\delta \in\left(0, T_{s}\right)$. However, in practical networked and embedded systems, the processing resources available for control calculations may vary and, at times, be insufficient to generate a control input within the prescribed time-delay $\delta$ [15]. In the sequel we will further develop our anytime control algorithm of [20], [22] to seek favorable trade-offs between processor 
and communication availability, and control performance. We will assume that the plant model (1) is globally stabilizable via state feedback.

Assumption 1 (Stabilizability): There exist $V: \mathbb{R}^{n} \rightarrow \mathbb{R}_{\geq 0}, \varphi_{1}, \varphi_{2} \in \mathscr{K}_{\infty}, \kappa: \mathbb{R}^{n} \rightarrow \mathbb{U}$, and a constant $\rho \in[0,1)$, such that

$$
\begin{array}{cc}
\varphi_{1}(|x|) \leq V(x) \leq \varphi_{2}(|x|), & \forall x \in \mathbb{R}^{n}, \\
V(f(x, \kappa(x))) \leq \rho V(x), & \forall x \notin \mathbb{B}_{d} .
\end{array}
$$

To encompass processing constraints, we will assume that the controller needs processor time to carry out mathematical computations, such as evaluating $\kappa$. However, input-output operations and simple operations at a bit level, e.g., writing data into buffers, shifting buffer contents and setting values to zero, do not require processor time.

Before proceeding we note that a direct implementation of $\kappa$ used in Assumption 1, when processing resources are time varying, sensor transmissions are event-triggered, and the sensor transmissions are affected by dropouts, results in the baseline event-based algorithm

$$
u(k)= \begin{cases}\kappa(x(k)) & \text { if } \beta(k)=1 \text { and processor is available, } \\ \mathbf{0}_{p} & \text { otherwise, }\end{cases}
$$

where the symbol $u(k)$ with $k \in \mathbb{N}_{0}$ denotes the plant input which is applied during the interval $\left[k T_{s}+\delta,(k+1) T_{s}+\delta\right)$. Whilst the baseline algorithm is intuitive, our previous works [20], [22] suggest that it will be outperformed by more elaborate control formulations.

\section{Event-Driven Anytime Control Algorithm}

The anytime algorithm is based on the following idea: control calculations are triggered whenever a new measurement is successfully received. However, the precise number of control inputs calculated depends on the processing resources available. At time intervals when the controller is provided with more processing resources than are needed to evaluate the current control input, the algorithm calculates a sequence of tentative future plant inputs. The sequence is stored in a local buffer and may be used when, at some future time steps, the processor availability precludes any control calculations even though new state information is received.

In our recent work [20], [22], we analyzed this algorithm for the simpler case where the controller has direct access to plant state $x(k)$ at all instants $k \in \mathbb{N}_{0}$. In the present work we alleviate this assumption by considering that sensor transmissions are event-triggered and through 


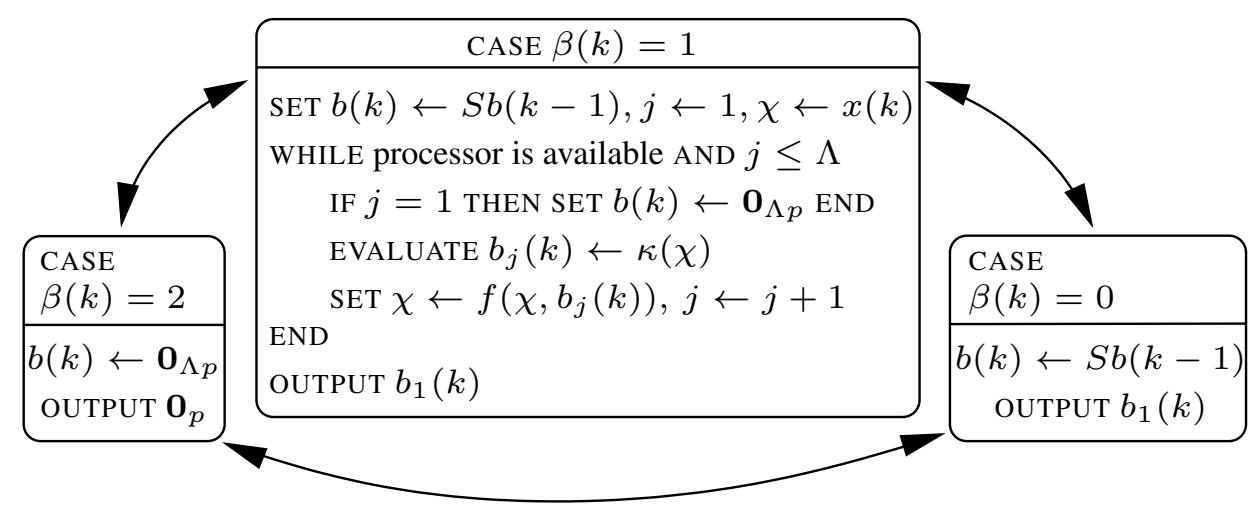

Fig. 2. Operating modes of anytime Algorithm $\mathrm{A}_{1}$ during the time interval $\left[k T_{s},(k+1) T_{s}\right)$.

a communication channel which introduces random dropouts. In addition, to save energy and processing resources, the controller is event-triggered. More precisely, the actions taken by the controller are guided by the value of $\beta(k)$ and the processor availability.

If $\beta(k)=1$, then the controller uses $x(k)$ to calculate tentative control values, provided the processor is available for control. This sequence will be stored in a buffer. If the processor is not available or $\beta(k)=0$, then the controller does not do any calculations and the plant input is provided by previously calculated buffered values (if available). The instances $\beta(k)=2$ refer to situations where the plant state is at the desired region $\mathbb{B}_{d}$, and $x(k)$ is not sent to the controller. In this scenario, the plant input is set to zero, the buffer is emptied, and the controller is switched off until the system state moves out of the desired region $\mathbb{B}_{d}$ and a new state measurement is received. Fig. 2 outlines the proposed algorithm. In this figure,

$$
S \triangleq\left[\begin{array}{ccccc}
0_{p} & I_{p} & 0_{p} & \ldots & 0_{p} \\
\vdots & \ddots & \ddots & \ddots & \vdots \\
0_{p} & \ldots \ldots & 0_{p} & I_{p} \\
0_{p} & \ldots \ldots \ldots & \ldots & 0_{p}
\end{array}\right] \in \mathbb{R}^{\Lambda p \times \Lambda p}, \quad b(k)=\left[\begin{array}{c}
b_{1}(k) \\
b_{2}(k) \\
\vdots \\
b_{\Lambda}(k)
\end{array}\right]
$$

where $\{b\}_{\mathbb{N}_{0}}$ denote the buffer states for a given buffer size $\Lambda \in \mathbb{N}$ and each $b_{j}(k) \in \mathbb{R}^{p}$, $j \in\{1, \ldots, \Lambda\}$.

For future use, we will denote by $N(k) \in\{0,1, \ldots, \Lambda\}$ the total number of iterations of the 
Step 1: At time $t=0$,

$$
\operatorname{SET} b(-1) \leftarrow \mathbf{0}_{\Lambda p}, k \leftarrow 0
$$

Step 2: IF $t \geq k T_{s}$,

THEN

SWITCH $\beta(k)$

CASE 2,

$$
\operatorname{SET} b(k) \leftarrow \mathbf{0}_{\Lambda p}, j \leftarrow 1 ;
$$

GOTO Step 4;

CASE 0,

SET $j \leftarrow 1, b(k) \leftarrow S b(k-1)$;

GOTO Step 4;

OTHERWISE

$$
\begin{aligned}
& \text { INPUT } x(k) ; \\
& \text { SET } \chi \leftarrow x(k), j \leftarrow 1, b(k) \leftarrow S b(k-1) ;
\end{aligned}
$$

END

END

Step 3: WHILE "sufficient processor time is available" and $j \leq \Lambda$ and time $t<(k+1) T_{s}$, EVALUATE $u_{j}(k)=\kappa(\chi)$;

IF $j=1$, THEN

$$
\begin{aligned}
& \text { OUTPUT } u_{1}(k) ; \\
& \text { SET } b(k) \leftarrow \mathbf{0}_{\Lambda p} ;
\end{aligned}
$$

END

$\operatorname{SET} b_{j}(k) \leftarrow u_{j}(k)$;

IF "sufficient processor time is not available" or $t \geq(k+1) T_{s}$, THEN GOTO Step 5;

END

$\operatorname{SET} \chi \leftarrow f\left(\chi, u_{j}(k)\right), j \leftarrow j+1$;

END

Step 4: IF $j=1$, THEN

$$
\text { OUTPUT } b_{1}(k) \text {; }
$$

END

Step 5: $\operatorname{SET} k \leftarrow k+1$ and GOTO Step 2;

Fig. 3. Algorithm $A_{1}$ 
while-loop which are carried out during the interval $t \in\left[k T_{s},(k+1) T_{s}\right)$. Thus, as described above, if $N(k) \geq 1$, then the entire sequence of tentative controls is $\left\{b_{1}(k), b_{2}(k), \ldots, b_{N(k)}(k)\right\}$ and the plant input is set to $b_{1}(k)$. If $N(k)=0$, then the plant input depends on the variable $\beta(k)$. If $\beta(k) \in\{0,1\}$ (i.e., $x(k)$ does not lie inside the desired region), then $u(k)$ is taken as the first $p$ elements of the shifted state $b(k)=S b(k-1)$. If, on the other hand, $\beta(k)=2$ indicating that $x(k) \in \mathbb{B}_{d}$, then the buffer is emptied and the plant input is set to zero, see Fig. 2.

Algorithm $\mathrm{A}_{1}$ amounts to a dynamic state feedback policy with internal state variable $b(k)$ which provides the plant input $u(k)$ and suggested plant inputs at future time steps. If new state information is received and more processor time is available, a longer trajectory of control inputs is calculated and stored in the buffer. If the buffer runs out of tentative plant inputs, then actuator values are set to zero. The algorithm does not require prior knowledge of future processor availability and hence can be employed in shared systems where the controller task can be preempted by other computational tasks at the processor.

\section{Stochastic Stability - Preliminaries}

For our subsequent analysis, it is convenient to investigate how many values in the state $b(k)$ stem from evaluating $\kappa, \ell \in \mathbb{N}_{0}$. As in [20], [22], we will refer to this value as the effective buffer length (at time $k$ ), and denote it as $\lambda(k) \in\{0,1, \ldots, \Lambda\}, k \in \mathbb{N}_{0}$ with $\lambda(-1)=0$. It is easy to see that for all $k \in \mathbb{N}_{0}$ we have

$$
\lambda(k)= \begin{cases}N(k) & \text { if } N(k) \geq 1, \\ \max \{0, \lambda(k-1)-1\}, & \text { if } N(k)=0 \text { and } \beta(k) \in\{0,1\}, \\ 0 & \text { if } \beta(k)=2 .\end{cases}
$$

To investigate stability, we make the following assumptions:

Assumption 2 (Processor availability): The sampling time of the plant (1) is such that processor availability for control at different time-instants is independent and identically distributed (i.i.d.). Thus, the process $\{N\}_{\mathbb{N}_{0}}$ has conditional probability distribution $p_{j} \triangleq \operatorname{Pr}\{N(k)=$ $j \mid \beta(k)=1\}$, where $p_{j} \in[0,1)$ are given and with $j \in\{0,1,2, \ldots, \Lambda\}$. For other realizations of $\beta(k)$, no plant inputs are calculated, thus, $\operatorname{Pr}\{N(k)=0 \mid \beta(k) \in\{0,2\}\}=1$.

Assumption 3 (Erasure channel): The binary transmission success process $\{\gamma\}_{\mathbb{N}_{0}}$ has conditional probabilities $\operatorname{Pr}\{\gamma(k)=1|| x(k) \mid \geq d\}=q, \operatorname{Pr}\{\gamma(k)=0|| x(k) \mid<d\}=1$. 
Assumption 4 (Open-loop bound): There exists $\alpha \geq \rho$ such that

$$
V\left(f\left(\chi, \mathbf{0}_{p}\right)\right) \leq \alpha V(\chi), \quad \forall \chi \in \mathbb{R}^{n} .
$$

where $\rho, V$ and $\varphi_{2}$ are as in (3). Further, $\mathbf{E}\left\{\varphi_{2}(|x(0)|)\right\}<\infty$.

It is worth noting that, by allowing for $\alpha>1$, Assumption 4 does not require that the open-loop system $x(k+1)=f\left(x(k), \mathbf{0}_{p}\right)$ be asymptotically stable. Further, note that Assumptions 1 and 4 are stated in terms of the same function $V$, see also [20, Section IV-A].

To go beyond stability and investigate stationarity, it is convenient to impose the following assumptions on the control policy $\kappa$

Assumption 5 (Continuity of $\kappa$ ): The control law $\kappa$ in (3) is such that $\kappa(x)=\mathbf{0}_{n}$ for all $x \in \mathbb{B}_{d}$ and $\kappa$ is continuous on $\mathbb{R}^{n}$.

\section{Stability with the Baseline Algorithm}

If the baseline algorithm is used and Assumption 2 holds, then

$$
x(k+1)= \begin{cases}f(x(k), \kappa(x(k))), & \text { if } N(k) \geq 1, \\ f\left(x(k), \mathbf{0}_{p}\right), & \text { if } N(k)=0 .\end{cases}
$$

The following result establishes conditions on system parameters which ensure that the closed loop (6) is stable in a stochastic sense.

Theorem 1 (Stability with baseline algorithm): Consider (6) and define $D \triangleq \varphi_{2}(d)$. Suppose that Assumptions 1 to 4 hold and that

$$
\Gamma \triangleq(1-q) \alpha+q\left(p_{0} \alpha+\left(1-p_{0}\right) \rho\right)<1,
$$

where $\rho \in[0,1)$ is the closed-loop bound in (3), $\alpha$ is the bound in (5), $q$ is the transmission success probability, and $p_{0}$ is the probability of the processor not being available for control. Then for all $x \in \mathbb{N}_{0}$,

$$
\mathbf{E}\left\{\varphi_{1}(|x(k)|)\right\} \leq \Gamma^{k} \mathbf{E}\left\{\varphi_{2}(x(0))\right\}+\frac{q\left(1-p_{0}\right)(\alpha-\rho) D}{1-\Gamma}<\infty .
$$

Proof: Note that, for i.i.d. processor and channel availabilities $\{x\}_{\mathbb{N}_{0}}$ in (6) is Markovian. This can be verified by noting that conditioning on $x(k)$ makes the event outcome $\beta(k)$ depend on $\gamma(k)$ only. To analyze stochastic stability using Lyapunov functions (see, e.g., [23]), we use the law of total expectation to write

$$
\mathbf{E}\{V(x(1)) \mid x(0)=\chi\}=\sum_{j=0}^{2} \mathbf{E}\{V(x(1)) \mid x(0)=\chi, \beta(0)=j\} \operatorname{Pr}\{\beta(0)=j \mid x(0)=\chi\} .
$$


If we now use (2), (3), (5) and the definition of $\mathbb{B}_{d}$, then:

$$
\begin{aligned}
& \mathbf{E}\{V(x(1)) \mid x(0)=\chi, \beta(0)=0\} \leq \alpha V(\chi) \\
& \mathbf{E}\{V(x(1)) \mid x(0)=\chi, \beta(0)=2\} \leq \alpha V(\chi)<\alpha \varphi_{2}(d),
\end{aligned}
$$

For $\beta(0)=1, x(0)$ is received. Using (5) and (6), we have

$$
\begin{gathered}
\mathbf{E}\{V(x(1)) \mid x(0)=\chi, \beta(0)=1\}=\sum_{j \in \mathbb{N}_{0}} \mathbf{E}\{V(x(1)) \mid x(0)=\chi, \beta(0)=1, N(0)=j\} \\
\times \operatorname{Pr}\{N(0)=j \mid x(0)=\chi, \beta(0)=1\} \leq\left(p_{0} \alpha+\left(1-p_{0}\right) \rho\right) V(\chi) .
\end{gathered}
$$

Now, if $x(0) \in \mathbb{B}_{d}$, then $\beta(0)=2$, thus $(\mathrm{V})$ and (8) provide

$$
\mathbf{E}\left\{V(x(1)) \mid x(0)=\chi \in \mathbb{B}_{d}\right\} \leq \alpha V(\chi)
$$

Further, since $\alpha-\Gamma=q\left(1-p_{0}\right)(\alpha-\rho)>0$ (see (7)) and $V(\chi)<D$ for all $\chi \in \mathbb{B}_{d}$, we have

$$
(\alpha-\Gamma) V(\chi)<(\alpha-\Gamma) D \Rightarrow \alpha V(\chi)<\Gamma V(\chi)+(\alpha-\Gamma) D, \quad \forall \chi \in \mathbb{B}_{d}
$$

On the other hand, if $x(0) \notin \mathbb{B}_{d}$, then (in view of Assumption 3), $\operatorname{Pr}\left\{\beta(0)=0 \mid x(0) \notin \mathbb{B}_{d}\right\}=$ $1-q$, and $\operatorname{Pr}\left\{\beta(0)=1 \mid x(0) \notin \mathbb{B}_{d}\right\}=q$. Thereby, substitution of (8) and (9) into (V) provides:

$$
\mathbf{E}\left\{V(x(1)) \mid x(0)=\chi \notin \mathbb{B}_{d}\right\} \leq \Gamma V(\chi) .
$$

Expressions (10)-(12) lead to:

$$
\mathbf{E}\{V(x(1)) \mid x(0)=\chi\}<\Gamma V(\chi)+(\alpha-\Gamma) D=\Gamma V(\chi)+q\left(1-p_{0}\right)(\alpha-\rho) D
$$

Consequently, Proposition 3.2 of [23], and (3) give

$$
\mathbf{E}\left\{\varphi_{1}(|x(k)|) \mid x(0)=\chi\right\} \leq \Gamma^{k} V(\chi)+\frac{q\left(1-p_{0}\right)(\alpha-\rho) D}{1-\Gamma},
$$

for all $k \in \mathbb{N}_{0}$. Using the law of total expectation and (3) yields the first inequality. The second follows from Assumption 4.

It is worth noting that whilst the condition (7) is independent of the size of $\mathbb{B}_{d}$, the ultimate bound is increasing in $d$. We can also consider two special cases. If $d=0$ and $q=1$, so that the sensor transmits at every instant $k \in \mathbb{N}_{0}$ and the communication channel does not introduce any dropouts, (7) reduces to $p_{0} \alpha+\left(1-p_{0}\right) \rho<1$, thus recovering our earlier result [20, Thm.1]. If the processor is available at every time-step (i.e., $p_{0}=0$ ), then the situation amounts to event-based 
control for non-linear systems using an erasure channel. In this case, the sufficient condition (7) becomes $(1-q) \alpha+\rho q<1$.

Theorem 2 (Stationarity with baseline algorithm): Consider (6), suppose that Assumptions 1 to 5 hold and that (7) holds. Then, there exists an invariant probability measure for $\{x\}_{\mathbb{N}_{0}}$. Furthermore, under every such invariant probability measure $\pi$,

$$
\mathbf{E}_{\pi}\left\{\varphi_{1}(|x|)\right\} \leq q\left(1-p_{0}\right)(\alpha-\rho) \varphi_{2}(d) /(1-\Gamma) .
$$

Proof: Let $\mathcal{P}\left(\mathbb{R}^{n}\right)$ denote the set of probability measures on $\mathbb{R}^{n}$ and define for every Borel $B, v_{T}(B)=(1 / T) \mathbf{E}\left\{\sum_{k=0}^{T-1} 1_{\{x(k) \in B\}}\right\}$, such that $v_{T} \in \mathcal{P}\left(\mathbb{R}^{n}\right)$ forms an expected empirical occupation measure sequence. We then have,

$$
\left\langle v_{T}, \varphi_{1}\right\rangle \triangleq \int v_{T}(d x) \varphi_{1}(|x|)=\frac{1}{T} \mathbf{E}\left\{\sum_{k=0}^{T-1} \varphi_{1}(|x(k)|)\right\} .
$$

Let $t_{0} \in \mathbb{N}$. By Theorem 1, we have that $\mathbf{E}\left\{\varphi_{1}(|x(k)|)\right\}$ and the subsequence $\left\{\left\langle v_{T}, \varphi_{1}\right\rangle, T \geq t_{0}\right\}$ are uniformly bounded by some $M_{t_{0}}<\infty$. Define $N_{r}:=\left\{x: \varphi_{1}(|x|) \leq r\right\}$. Since $\varphi_{1}$ is monotone and unbounded, by an application of Markov's inequality, we have

$$
M_{t_{0}} \geq \int v_{T}(d x) \varphi_{1}(|x|) \geq \int_{\mathbb{X} \backslash N_{r}} v_{T}(d x) \varphi_{1}(|x|) \geq r v_{T}\left(\mathbb{R}^{n} \backslash N_{r}\right) .
$$

Thus, $v_{T}\left(N_{r}\right) \geq 1-M_{t_{0}} / r$, and hence for every $\epsilon=M_{t_{0}} / r>0$, there exists a compact set $N_{M_{t_{0}} / \epsilon}=\left\{x: \varphi_{1}(|x|) \leq M_{t_{0}} / \epsilon\right\}$ such that $v_{t}\left(N_{M_{t_{0}} / \epsilon}\right) \geq 1-\epsilon$. The sequence $\left\{v_{t}, t \geq t_{0}\right\}$ is, hence, a tight sequence with a converging subsequence $v_{t_{k}}$ converging to some $v^{*} \in \mathcal{P}\left(\mathbb{R}^{n}\right)$. By (6), if $x(t) \in \mathbb{B}_{d}$ the control action is zero and outside $\mathbb{B}_{d}$, either zero control is applied or $\kappa(x(t))$ is applied. Since $\kappa$ is continuous and is zero inside $\mathbb{B}_{d}$ (see Assumption 5), the Markov chain is weak Feller. ${ }^{1}$ Consequently, it can be shown that every limit of such a subsequence is invariant (see, e.g., [24, Ch. 12]) and satisfies $\left\langle v_{T}, \varphi_{1}\right\rangle \leq M_{t_{0}}$. By Theorem 1, by increasing $t_{0}$, $M_{t_{0}}$ can be taken to be arbitrarily close to $q\left(1-p_{0}\right)(\alpha-\rho) \varphi_{2}(d) /(1-\Gamma)$.

\section{Stability With the Anytime Algorithm}

The analysis of the event-based anytime algorithm is more involved than that of the baseline system (6). First, due to buffering, $\{x\}_{\mathbb{N}_{0}}$ will in general not be a Markov process. Further, the

\footnotetext{
${ }^{1} \mathrm{~A}$ Markov chain $\{x(k)\}_{k \in \mathbb{N}_{0}}$ is (weak) Feller if $\mathbf{E}\{h(x(k+1)) \mid x(k)=\chi\}$ is continuous in $\chi$, for every continuous and bounded function $h$.
} 
distribution of $\{\beta\}_{\mathbb{N}_{0}}$ is difficult to derive for general plant models. This makes the approaches of [20], [22] insufficient to treat the present case.

For ease of exposition, we assume that the initial effective buffer length, $\lambda(0)=0$, and denote the time steps where $\lambda(k)=0$ via $\mathcal{K}=\left\{k_{i}\right\}_{i \in \mathbb{N}_{0}}$, where $k_{0}=0$ and $k_{i+1}=\inf \{k \in \mathbb{N}: k>$ $\left.k_{i}, \lambda(k)=0\right\}, i \in \mathbb{N}_{0}$. We also describe the amount of time steps between consecutive elements of $\mathcal{K}$ via the process $\left\{\Delta_{i}\right\}_{i \in \mathbb{N}_{0}}$, where $\Delta_{i} \triangleq k_{i+1}-k_{i}$. It is easy to see that

$$
\beta\left(k_{i}+\ell\right) \in\{0,1\}, \quad \forall \ell \in\left\{1,2, \ldots, \Delta_{i}-1\right\}, \quad \forall i \in \mathbb{N}_{0}
$$

whereas $\beta\left(k_{i}\right) \in\{0,1,2\}, \forall i \in \mathbb{N}_{0}$ and $x\left(k^{*}\right) \in \mathbb{B}_{d} \Rightarrow k^{*} \in \mathcal{K}$. In contrast to the cases examined in [20], [22], due to the event-triggering mechanism, $\left\{\Delta_{i}\right\}_{i \in \mathbb{N}_{0}}$ is, in general, not i.i.d. In fact, the distribution of $\Delta_{i}$ depends on $x\left(k_{i}\right)$ and is difficult to characterize. To study stability of the event-based anytime algorithm, we will develop a state-dependent random-time drift condition. Our first result, states that whilst $\{x\}_{\mathbb{N}_{0}}$ is in general not Markovian, the state sequence at the time steps $k_{i} \in \mathcal{K}$, is a Markov process.

Lemma 1 (Markov property of the sampled process): Consider (1) controlled via Algorithm $\mathrm{A}_{1}$ and suppose that Assumptions 2 and 3 hold. Then $\{x\}_{\mathcal{K}}$ is Markovian.

Proof: The definition of $\mathcal{K}$ gives that $\forall k_{i} \in \mathcal{K}$ we have $u\left(k_{i}\right)=\mathbf{0}_{p}, b\left(k_{i}\right)=\mathbf{0}_{\Lambda p}, \lambda\left(k_{i}\right)=$ $N\left(k_{i}\right)=0$. Thus, the plant state at time $k_{i+1}$ depends only on $x\left(k_{i}\right)$ and the sample paths $\left\{N\left(k_{i}+1\right), N\left(k_{i}+2\right), \ldots, N\left(k_{i+1}-1\right)\right\}$ and $\left\{\gamma\left(k_{i}+1\right), \gamma\left(k_{i}+2\right), \ldots, \gamma\left(k_{i+1}-1\right)\right\}$. The result follows since $\{N\}_{\mathbb{N}_{0}}$ and $\{\gamma\}_{\mathbb{N}_{0}}$ are i.i.d.

The following result provides a sufficient condition for stochastic stability of the closed loop when the event-based anytime control algorithm of Section III is used over an erasure channel.

Theorem 3 (Stability with Algorithm $A_{1}$ ): Suppose that Assumptions 1 to 4 hold and define

$$
\Omega \triangleq \alpha \sum_{j \in \mathbb{N}} \rho^{j-1} \operatorname{Pr}\left\{\Delta_{i}=j \mid \beta\left(k_{i+1}\right) \neq 2\right\} .
$$

If Algorithm $\mathrm{A}_{1}$ is used and $\Omega<1$, then

$$
\max _{k \in\left\{k_{i}, k_{i}+1, \ldots, k_{i+1}-1\right\}} \mathbf{E}\left\{\varphi_{1}(|x(k)|)\right\} \leq \frac{1+\alpha-\rho}{1-\rho} \Omega^{i} \mathbf{E}\left\{\varphi_{2}(x(0))\right\}+\frac{\varphi_{2}(d)}{1-\Omega}<\infty, \quad \forall i \in \mathbb{N} .
$$

Proof: We first note that for all $k_{i} \in \mathcal{K}$ and $\ell \in\left\{1, \ldots, \Delta_{i}-1\right\}, u\left(k_{i}\right)=\mathbf{0}_{p}$ and $u\left(k_{i}+\ell\right)=$ $\kappa\left(x\left(k_{i}+\ell\right)\right)$. Therefore, the function $V\left(x\left(k_{i+1}\right)\right)$ can be bounded by using (3) and (5), leading to

$$
\mathbf{E}\left\{V\left(x\left(k_{i+1}\right)\right) \mid x\left(k_{i}\right)=\chi, \Delta_{i}=j\right\} \leq \alpha \rho^{j-1} V(\chi), \forall \chi \in \mathbb{R}^{n} .
$$


To account for event-based transmission, we consider instances where the buffer is emptied triggered by $\beta(k)=2$. At these instances, (16) holds; further, $V\left(k_{i+1}\right)<D \triangleq \varphi_{2}(d)$. Thus,

$$
\mathbf{E}\left\{V\left(x\left(k_{i+1}\right)\right) \mid x\left(k_{i}\right)=\chi, \Delta_{i}=j, \beta\left(k_{i+1}\right)=2\right\}<D, \quad \forall j \in \mathbb{N} .
$$

By using the law of total expectation twice, we thus obtain,

$$
\begin{aligned}
& \mathbf{E}\left\{V\left(x\left(k_{i+1}\right)\right) \mid x\left(k_{i}\right)=\chi\right\}=\mathbf{E}\left\{V\left(x\left(k_{i+1}\right)\right) \mid x\left(k_{i}\right)=\chi, \beta\left(k_{i+1}\right)=2\right\} \operatorname{Pr}\left\{\beta\left(k_{i+1}\right)=2 \mid x\left(k_{i}\right)=\chi\right\} \\
& +\mathbf{E}\left\{V\left(x\left(k_{i+1}\right)\right) \mid x\left(k_{i}\right)=\chi, \beta\left(k_{i+1}\right) \neq 2\right\} \operatorname{Pr}\left\{\beta\left(k_{i+1}\right) \neq 2 \mid x\left(k_{i}\right)=\chi\right\} \\
& \leq D+\mathbf{E}\left\{V\left(x\left(k_{i+1}\right)\right) \mid x\left(k_{i}\right)=\chi, \beta\left(k_{i+1}\right) \neq 2\right\} \\
& =D+\sum_{j \in \mathbb{N}} \mathbf{E}\left\{V\left(x\left(k_{i+1}\right)\right) \mid x\left(k_{i}\right)=\chi, \beta\left(k_{i+1}\right) \neq 2, \Delta_{i}=j\right\} \operatorname{Pr}\left\{\Delta_{i}=j \mid x\left(k_{i}\right)=\chi, \beta\left(k_{i+1}\right) \neq 2\right\} \\
& \leq D+\sum_{j \in \mathbb{N}} \alpha \rho^{j-1} V(\chi) \operatorname{Pr}\left\{\Delta_{i}=j \mid x\left(k_{i}\right)=\chi, \beta\left(k_{i+1}\right) \neq 2\right\}=D+\Omega V(\chi), \forall \chi \in \mathbb{R}^{n}
\end{aligned}
$$

with $\Omega$ as in (14) and where, to derive the last equality, we have used Assumption 2. Since $\{x\}_{\mathcal{K}}$ is Markovian, [23, Prop. 3.2] yields that $\Omega<1$ guarantees

$$
\mathbf{E}\left\{V\left(x\left(k_{i}\right)\right) \mid x\left(k_{0}\right)=\chi\right\} \leq \Omega^{i} V(\chi)+\frac{D}{1-\Omega}, \quad \forall i \in \mathbb{N}_{0} .
$$

Now, since (16) holds, by a method similar to the one used in the proof of [22, Thm.1], we can establish the (admittedly loose) bound:

$$
\mathbf{E}\left\{\sum_{k=k_{i}}^{k_{i+1}-1} V(x(k)) \mid x\left(k_{0}\right)=\chi\right\} \leq \frac{1+\alpha-\rho}{1-\rho} \Omega^{i} V(\chi)+\frac{D}{1-\Omega}, \quad \forall i \in \mathbb{N} .
$$

Using the law total expectation, (3) and Assumption 4 gives (15).

The above result establishes a sufficient condition for the system to be stochastically stable. The quantity (14) is stated in terms of a conditional distribution of $\Delta_{i}$, which can be characterized as follows:

Lemma 2 (Conditional distribution of $\Delta_{i}$ ): Suppose that Assumptions 2 and 3 hold and that Algorithm $\mathrm{A}_{1}$ is used. We then have

$$
\frac{\operatorname{Pr}\left\{\Delta_{i}=j \mid \beta\left(k_{i+1}\right) \neq 2\right\}}{1-q+p_{0} q}=\left\{\begin{array}{ll}
1 & \text { if } j=1, \\
\theta^{T} G^{j-2} e_{1} & \text { if } j \geq 2,
\end{array} \quad \forall(i, j) \in \mathbb{N}_{0} \times \mathbb{N}\right.
$$

where $\theta^{T}=q\left[\begin{array}{lll}p_{1} & \ldots & p_{\Lambda}\end{array}\right]$ and $e_{1}^{T}=\left[\begin{array}{llll}1 & 0 & \ldots & 0\end{array}\right]$. In (20), the entries of the matrix $G=\left[g_{\ell j}\right], \ell, j \in\{1,2, \ldots, \Lambda\}$ are $g_{\ell j}=p_{j} q, \forall(\ell, j) \in\{3,4, \ldots, \Lambda\} \times\{1,2, \ldots, \ell-2\} \cup$ $\{1,2, \ldots, \Lambda\} \times\{\ell, \ell+1, \ldots, \Lambda\} ;$ and $g_{\ell(\ell-1)}=1-q+\left(p_{0}+p_{\ell-1}\right) q, \forall \ell \in\{2,3, \ldots, \Lambda\}$. 
Proof: We first note that our focus is on the time sequences of the form $\mathcal{I}_{i} \triangleq\left\{k_{i}+\right.$ $\left.1, \ldots, k_{i+1}\right\}$ where $k_{i} \in \mathcal{K}, i \in \mathbb{N}_{0}$ and where $\beta(k) \neq 2, \forall k \in \mathcal{I}_{i}$. Given Assumptions 2 and 3 and the buffering mechanism described in Section III, it follows that $\{\lambda(k)\}$ during every interval $k \in \mathcal{I}_{i}, i \in \mathbb{N}_{0}$, is a homogeneous Markov Chain. The process $\Delta_{i}$ then amounts to the first return times to 0 of this finite Markov Chain. To characterize the latter, we need to evaluate the transition probabilities $g_{\ell j} \triangleq \operatorname{Pr}\left\{\lambda(k+1)=j \mid \lambda(k)=\ell, k \in \mathcal{I}_{i}, k+1 \in \mathcal{I}_{i}\right\}$. Without loss of generality, we will set $k=0$. We begin by considering transitions from $\ell \in\{0,1\}$ to 0 :

$$
\begin{aligned}
g_{\ell 0}= & \operatorname{Pr}\{N(1)=0 \mid \beta(1)=0\} \operatorname{Pr}\{\beta(1)=0 \mid \beta(1) \neq 2\} \\
& +\operatorname{Pr}\{N(1)=0 \mid \beta(1)=1\} \operatorname{Pr}\{\beta(1)=1 \mid \beta(1) \neq 2\}=(1-q)+p_{0} q, \quad \forall \ell \in\{0,1\} .
\end{aligned}
$$

For $\ell \in\{2,3, \ldots, \Lambda\}$, we have $g_{\ell 0}=0$. The buffer length diminishes by one for the scenarios considered below:

$$
\begin{aligned}
g_{\ell(\ell-1)}= & \operatorname{Pr}\{N(1)=0 \mid \beta(1)=0\} \operatorname{Pr}\{\beta(1)=0 \mid \beta(1) \neq 2\}+\operatorname{Pr}\{N(1)=0 \mid \beta(1)=1\} \\
& \times \operatorname{Pr}\{\beta(1)=1 \mid \beta(1) \neq 2\}+\operatorname{Pr}\{N(1)=\ell-1 \mid \beta(1)=1\} \operatorname{Pr}\{\beta(1)=1 \mid \beta(1) \neq 2\} \\
= & (1-q)+p_{0} q+p_{\ell-1} q, \quad \forall \ell \in\{2,3, \ldots, \Lambda\} .
\end{aligned}
$$

The other transitions are related to when $\lambda(k+1)=N(k+1)$, for $(\ell, j) \in\{\{3,4, \ldots, \Lambda\} \times$ $\{1,2, \ldots, \ell-2\}\} \cup\{\{1,2, \ldots, \Lambda\} \times\{\ell, \ell+1, \ldots, \Lambda\}\} \cup\{0 \times\{1,2, \ldots, \Lambda\}\}$. Here we have:

$$
\begin{aligned}
g_{\ell j}= & \operatorname{Pr}\{\lambda(1)=j \mid \lambda(0)=\ell, \beta(1)=0\} \operatorname{Pr}\{\beta(1)=0 \mid \beta(1) \neq 2\} \\
& +\operatorname{Pr}\{\lambda(1)=j \mid \lambda(0)=\ell, \beta(1)=1\} \operatorname{Pr}\{\beta(1)=1 \mid \beta(1) \neq 2\} \\
= & \operatorname{Pr}\{N(1)=j \mid \beta(1)=0\} \operatorname{Pr}\{\beta(1)=0 \mid \beta(1) \neq 2\}+\operatorname{Pr}\{N(1)=j \mid \beta(1)=1\} \\
& \times \operatorname{Pr}\{\beta(1)=1 \mid \beta(1) \neq 2\}=0(1-q)+p_{j} q=p_{j} q .
\end{aligned}
$$

The derivation of (20) now follows as in [22, Lemma 2] by setting up a recursion on the first passage time of state $\ell \in\{1, \ldots, \Lambda\}$ to 0 and then considering the transitions away from 0 .

As a consequence of Lemma $2, \Omega$ in (14) can be written as:

$$
\Omega=\alpha\left(1-q+p_{0} q\right)\left(1+\rho \theta^{T}(I-\rho G)^{-1} e_{1}\right)
$$

and the stability condition in Theorem $3, \Omega<1$, becomes

$$
\left[\begin{array}{lll}
p_{1} & \ldots & p_{\Lambda}
\end{array}\right]\left(I_{\Lambda}-\rho G\right)^{-1} e_{1}<\frac{1-\alpha+\alpha q\left(1-p_{0}\right)}{\alpha \rho q\left(1-q\left(1-p_{0}\right)\right)},
$$

which is independent of the size of $\mathbb{B}_{d}$. 
Sufficient conditions for stationarity can be stated as follows:

Theorem 4 (Stationarity with Algorithm $A_{1}$ ): Suppose that Assumptions 1 to 5 hold. If Algorithm $\mathrm{A}_{1}$ is used and $\Omega<1$, then there exists an invariant probability measure for $\{x\}_{\mathcal{K}}$ as well as for the aggregated Markov process, $\left\{x_{[k, k-(\Lambda-1)]}\right\}_{k \in \mathbb{N}}$, where

$$
x_{[k, k-(\Lambda-1)]} \triangleq\{x(k), x(k-1), \cdots, x(k-\Lambda+1)\} .
$$

Furthermore, under every invariant probability measure $\pi, \mathbf{E}_{\pi}\{V(x)\}<\varphi_{2}(d) /(1-\Omega)$.

Proof: First note that if $N(k) \geq 1$, then $u(k)$ is determined by the current state. If the processor is not available, then either $u(k)$ has been determined by the states which are at most $\Lambda$ time stages old, or $u(k)=\mathbf{0}_{p}$. Since the processor availability is independent of the state, the stochastic process $\left\{x_{[k, k-\Lambda+1]}\right\}$ is Markovian. Let $\mathbf{z}(k) \triangleq x_{[k, k-\Lambda+1]}$. From Assumption 5, $\{\mathbf{z}\}_{\mathbb{N}_{0}}$ is also weak Feller.

We first invoke Theorem 2.1 in [25] with $\mathcal{K}$ containing the sequence of stopping times. Since

$$
\mathbf{E}\left\{V\left(x\left(k_{i+1}\right)\right) \mid x\left(k_{i}\right)=\chi\right\} \leq V(\chi)-(1-\Omega) V(\chi)+D, \quad \forall \chi \in \mathbb{R}^{n},
$$

and the sampled chain is weak Feller, it follows that $\{x\}_{\mathcal{K}}$ admits an invariant probability measure.

Define $\tilde{V}(\mathbf{z}(k)) \triangleq V(x(k))$. Now, note that by (18), with $\Omega<1, \mathbf{E}\left\{\tilde{V}\left(\mathbf{z}\left(k_{i+1}\right)\right) \mid \mathbf{z}\left(k_{i}\right)=\right.$ $\chi\} \leq D+\Omega \tilde{V}(\chi), \forall \chi$. Thus, $\mathbf{E}\left\{\tilde{V}\left(\mathbf{z}\left(k_{i+1}\right)\right) \mid \mathbf{z}\left(k_{i}\right)=\chi\right\} \leq \tilde{V}(\chi)-(1-\Omega) \tilde{V}(\chi)+D, \forall \chi$, and since $V$ is monotone increasing and by Assumption 4, there exists a compact set $\mathcal{S}$ such that for $1-\Omega>\zeta>0, \mathbf{E}\left\{V\left(x\left(k_{i+1}\right)\right) \mid x\left(k_{i}\right)=x\right\} \leq V(x)-\zeta V(x)+D 1_{x \in \mathcal{S}}, \quad \forall x \in \mathbb{R}^{n}$. Since $V(x(t))$ is bounded from below outside $\mathbb{B}_{d}$, and $x(k) \notin \mathbb{B}_{d}$ for $k \notin \mathcal{K}$, and that (19) implies that for some $M_{1}<\infty$

$$
\mathbf{E}\left\{\sum_{k=k_{i}}^{k_{i+1}-1} V(x(k)) \mid x\left(k_{0}\right)=\chi\right\} \leq M_{1}
$$

it follows that $\sup _{x_{k_{i}}} \mathbf{E}\left\{k_{i+1}-k_{i} \mid x_{k_{i}}\right\}<\infty$. Finally, by Assumption 4, if $x_{t} \in \mathcal{S}$ then $x_{[t+\Lambda-1, t]} \in$ $\overline{\mathcal{S}}$ where $\overline{\mathcal{S}}$ is a compact set. Thus, Theorem 2.2 in [25] implies that there exists an invariant probability distribution, $\pi$, for $\{\mathbf{z}\}_{\mathbb{N}_{0}}$.

Since (21) holds, with $P^{m} V(\chi):=\mathbf{E}\left\{V\left(x\left(k_{m}\right)\right) \mid x\left(k_{0}\right)=\chi\right\}$, following arguments similar to the proof of Theorem 2.2 of [25], for every realization of $x\left(k_{0}\right)$, it follows that

$$
(1-\Omega) \limsup _{T \rightarrow \infty} \frac{1}{T} \mathbf{E}\left\{\sum_{i=0}^{T-1} V\left(x\left(k_{i}\right)\right)\right\} \leq \limsup _{T \rightarrow \infty} \frac{1}{T}\left(V\left(x\left(k_{0}\right)\right)+\sum_{i=0}^{T-1} D\right) .
$$




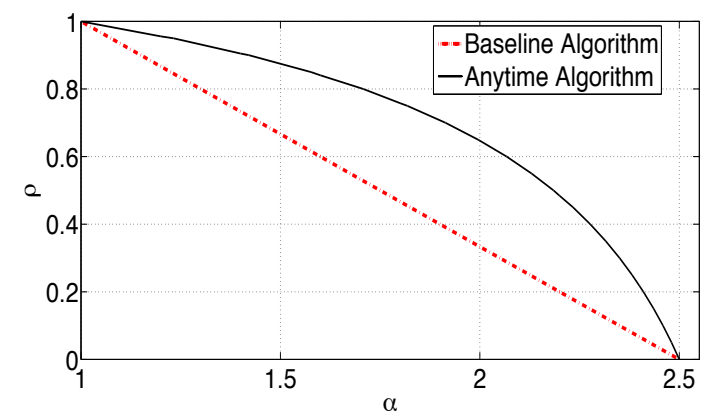

Fig. 4. Boundaries of stability: $\Omega=1$ (solid line) and $\Gamma=1$ (dashed).

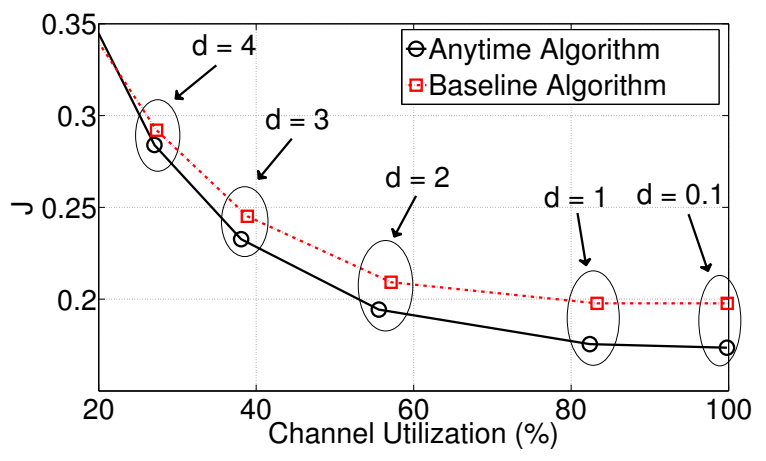

Fig. 5. Empirical cost versus channel utilization for different values of $d$.

Thus, $\lim \sup _{T \rightarrow \infty}(1 / T) \sum_{m=0}^{T-1} P^{m} V\left(x\left(k_{m}\right)\right) \leq D /(1-\Omega)$. Applying Fatou's lemma, we obtain $\limsup _{T \rightarrow \infty} \mathbf{E}_{\pi}\left\{\frac{1}{T} \sum_{i=0}^{T-1} \min \left(N, V\left(x\left(k_{i}\right)\right)\right)\right\} \leq \mathbf{E}_{\pi}\left\{\limsup _{T \rightarrow \infty} \frac{1}{T} \sum_{i=0}^{T-1} \min \left(N, V\left(x\left(k_{i}\right)\right)\right)\right\} \leq \frac{D}{1-\Omega}$.

Then, by the monotone convergence theorem, by letting $N \rightarrow \infty$,

$$
\limsup _{T \rightarrow \infty} \mathbf{E}_{\pi}\left\{\frac{1}{T} \sum_{i=0}^{T-1} V\left(x\left(k_{i}\right)\right)\right\} \leq \frac{D}{1-\Omega} .
$$

Thus, there exists an invariant probability measure both for the original chain and for the sampled chain; under every such invariant probability measure $\pi, \mathbf{E}_{\pi}\{V(x)\}<D /(1-\Omega)$.

\section{NUMERICAL EXAMPLES}

We first compare the stability conditions derived for a specific case. Suppose that the buffer length is given by $\Lambda=4$, whereas $p_{i}=0.2, i \in\{0, \ldots, 4\}$, and $q=0.75$. The stability region boundaries, see (7) and (14), in terms of $\alpha$ and $\rho$ are depicted in Fig. 4. It can be seen that 
the guaranteed stable region (under the curve) provided by our results is larger when using Algorithm $\mathrm{A}_{1}$ than when using (4).

Next, we consider an open-loop unstable constrained plant model of the form (1), but with additive noise:

$$
\left[\begin{array}{c}
x_{1}(k+1) \\
x_{2}(k+1)
\end{array}\right]=\left[\begin{array}{c}
x_{2}(k)+u_{1}(k) \\
-\operatorname{sat}\left(x_{1}(k)+x_{2}(k)\right)+u_{2}(k)
\end{array}\right]+\left[\begin{array}{c}
w_{1}(k) \\
w_{2}(k)
\end{array}\right]
$$

where

$$
\operatorname{sat}(\mu)= \begin{cases}-10, & \text { if } \mu<-10, \\ \mu & \text { if } \mu \in[-10,10], \\ 10, & \text { if } \mu>10,\end{cases}
$$

see [20, Example 2]. The initial condition $x(0)$ and the disturbance $w(k)$ are zero-mean i.i.d.

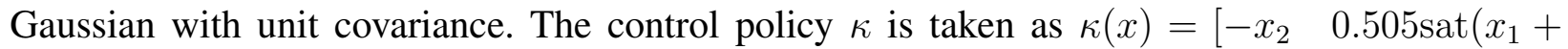
$\left.\left.x_{2}\right)\right]^{T}, x \in \mathbb{R}^{2}$. If we choose $V(x)=2|x|$, then direct calculations give that

$$
\begin{aligned}
& V(f(x, \kappa(x)))=0.99\left|\operatorname{sat}\left(x_{1}+x_{2}\right)\right| \leq 0.99\left|x_{1}+x_{2}\right| \\
& \quad \leq 1.98 \max \left\{\left|x_{1}\right|,\left|x_{2}\right|\right\}-\max \left\{\left|x_{1}\right|,\left|x_{2}\right|\right\}+|x| \leq 1.98|x| .
\end{aligned}
$$

Thus, Assumption 1 holds with $\rho=0.99$, and $\varphi_{1}(s)=\varphi_{2}(s)=2 s$. Processor availability and $\Lambda$ are taken as above, but we now set $q=0.4$. Performance is evaluated through the empirical cost $J \triangleq \frac{1}{50}\left(\sum_{k=0}^{49}|x(k)|^{2}\right)$ and the Channel Utilization (\%), calculated as

$\frac{\text { Total number of time steps at which } \beta(k) \neq 2}{\text { Total number of time steps }}(\%)$.

By averaging over $10^{4}$ realizations, Fig. 5 is obtained. As can be seen in that figure, the proposed event-based anytime control algorithm gives better trade-offs between empirical cost and channel utilization.

\section{CONCLUSIONS}

This work considered the control of a non-linear process with both communication and processing constraints. A sensor node transmits data to the controller across a channel that stochastically erases data. The control algorithm is executed over a processor that can provide only limited, time-varying and a priori unknown processing resources. To reduce the communication frequency, the sensor utilizes an event-triggered scheme. Similarly, to better utilize the processor availability, 
the control input is calculated by using an anytime control algorithm. For the resulting system, we present stochastic stability and stationarity results. Numerical studies illustrate that significant performance gains can be obtained by using the proposed algorithm. Future work includes the extension of the analysis to noisy systems, and establishing further stability properties such as ergodicity and rates of convergence to equilibrium.

\section{REFERENCES}

[1] V. Gupta, A. F. Dana, J. P. Hespanha, R. M. Murray, and B. Hassibi, "Data transmission over networks for estimation and control," IEEE Trans. Automat. Contr., vol. 54, pp. 1807-1819, Aug. 2009.

[2] O. C. Imer, S. Yüksel, and T. Bassar, "Optimal control of LTI systems over unreliable communication links," Automatica, vol. 42, pp. 1429-1439, Sept. 2006.

[3] L. Schenato, B. Sinopoli, M. Franceschetti, K. Poolla, and S. S. Sastry, "Foundations of control and estimation over lossy networks," Proc. IEEE, vol. 95, pp. 163-187, Jan. 2007.

[4] D. E. Quevedo and D. Nešić, "Robust stability of packetized predictive control of nonlinear systems with disturbances and Markovian packet losses," Automatica, vol. 48, pp. 1803-1811, Aug. 2012.

[5] L. Li, M. Lemmon, and X. Wang, "Event-triggered state estimation in vector linear processes," in Proc. Amer. Contr. Conf., pp. 2138-2143, 2010.

[6] P. Tabuada, "Event-triggered real-time scheduling of stabilizing control tasks," IEEE Trans. Automat. Contr., vol. 52, pp. 1680-1685, Sept. 2007.

[7] Y. Xu and J. Hespanha, "Optimal communication logics in networked control systems," in Proc. IEEE Conf. Decis. Contr., pp. 3527-3532, 2004.

[8] C. Ramesh, H. Sandberg, and K. H. Johansson, "Steady state performance analysis of multiple state-based schedulers with CSMA," in Proc. IEEE Conf. Decis. Contr., 2011.

[9] M. Xia, V. Gupta, and P. J. Antsaklis, "Networked state estimation over a shared communication medium," in Proc. Amer. Contr. Conf., 2013.

[10] M. Rabi and K. H. Johansson, "Scheduling packets for event-triggered control," in Proc. Europ. Contr. Conf., pp. 37793784, 2009.

[11] R. Blind and F. Allgöwer, "Analysis of networked event-based control with a shared communication medium: Part 1 pure aloha," in Proc. IFAC World Congr., 2011.

[12] A. Cervin and T. Henningsson, "Scheduling of event-triggered controllers on a shared network," in Proc. IEEE Conf. Decis. Contr., pp. 3601-3606, 2008.

[13] L. K. McGovern and E. Feron, "Closed-loop stability of systems driven by real-time dynamic optimization algorithms," in Proc. IEEE Conf. Decis. Contr., vol. 4, (Phoenix, AZ), pp. 3690-3696, Dec. 1999.

[14] D. Henriksson and J. Åkesson, "Flexible implementation of model predictive control using sub-optimal solutions," Tech. Rep. Internal Report No. TFRT-7610-SE, Dep. of Automatic Control, Lund University, 2004.

[15] P. Andrianiaina, A. Seuret, and D. Simon, "Robust system control method with short execution deadlines." European Patent Application EP 2568346 A1, Airbus Operations Toulouse, March 2013.

[16] A. Cervin, M. Velasco, P. Martí, and A. Camacho, "Optimal online sampling period assignment: Theory and experiments," IEEE Trans. Contr. Syst. Technol., vol. 18, June 2010.

[17] R. Bhattacharya and G. J. Balas, "Anytime control algorithms: Model reduction approach," AIAA Journal of Guidance, Control and Dynamics, vol. 27, pp. 767-776, Sept.-Oct. 2004.

[18] L. Greco, D. Fontanelli, and A. Bicchi, "Almost sure stability of anytime controllers via stochastic scheduling," in Proc. IEEE Conf. Decis. Contr., (New Orleans, LA), pp. 5640-5645, Dec. 2007. 
[19] V. Gupta and F. Luo, "On a control algorithm for time-varying processor availability," IEEE Trans. Automat. Contr., vol. 58, Mar. 2013.

[20] D. E. Quevedo and V. Gupta, "Sequence-based anytime control," IEEE Trans. Automat. Contr., vol. 58, pp. 377-390, Feb. 2013.

[21] C. Ramesh, H. Sandberg, and K. H. Johansson, "On the dual effect in state-based scheduling of networked control systems," in Proc. Amer. Contr. Conf., pp. 2216-2221, 2011.

[22] D. E. Quevedo and V. Gupta, "Stability of sequence-based anytime control with Markovian processor availability," in Proc. Austr. Contr. Conf., 2011.

[23] S. P. Meyn, "Ergodic theorems for discrete time stochastic systems using a stochastic Lyapunov function," SIAM Journal on Control and Optimization, vol. 27, pp. 1409-1439, Nov. 1989.

[24] S. Meyn and R. L. Tweedie, Markov Chains and Stochastic Stability. Cambridge University Press, 2009.

[25] S. Yüksel and S. P. Meyn, "Random-time, state-dependent stochastic drift for Markov chains and application to stochastic stabilization over erasure channels," IEEE Trans. Automat. Contr., vol. 58, no. 1, pp. 47-59, 2013. 